\title{
MALLIAVIN CALCULUS METHOD FOR ASYMPTOTIC EXPANSION OF DUAL CONTROL PROBLEMS
}

\author{
MICHAEL MONOYIOS \\ MATHEMATICAL INSTITUTE \\ UNIVERSITY OF OXFORD \\ ACCEPTED FOR PUBLICATION IN SIAM JOURNAL ON FINANCIAL MATHEMATICS
}

\begin{abstract}
We develop a technique based on Malliavin-Bismut calculus ideas, for asymptotic expansion of dual control problems arising in connection with exponential indifference valuation of claims, and with minimisation of relative entropy, in incomplete markets. The problems involve optimisation of a functional of Brownian paths on Wiener space, with the paths perturbed by a drift involving the control. In addition there is a penalty term in which the control features quadratically. The drift perturbation is interpreted as a measure change using the Girsanov theorem, leading to a form of the integration by parts formula in which a directional derivative on Wiener space is computed. This allows for asymptotic analysis of the control problem. Applications to incomplete Itô process markets are given, in which indifference prices are approximated in the low risk aversion limit. We also give an application to identifying the minimal entropy martingale measure as a perturbation to the minimal martingale measure in stochastic volatility models.
\end{abstract}

\section{INTRODUCTION}

In this article we use an approach to the Malliavin calculus, pioneered by Bismut [6], in which perturbations to Brownian paths on Wiener space are interpreted as measure changes via the Girsanov theorem, to derive asymptotic expansions for certain entropy-weighted stochastic control problems. These problems typically arise from the dual to investment and indifference pricing problems under exponential utility.

In the dual approach to investment and hedging problems in incomplete markets, optimisation problems over trading strategies are converted to optimisations over probability measures. For example, in exponential indifference pricing of a European claim with payoff $F$, the dual control representation of the indifference price is to maximise the expectation of the payoff subject to an entropic penalty involving the risk aversion $\alpha$ (as we show in Lemma 4.8). In an Itô process setting, the optimisation over measures leads to a problem in which the control is a drift perturbation to a multi-dimensional Brownian motion. This leads us to consider control problems of the form (with $\|\cdot\|$ denoting the Euclidean norm)

$$
\sup _{\varphi} \mathbb{E}\left[F\left(W+\varepsilon \int_{0}^{\cdot} \varphi_{s} \mathrm{~d} s\right)-\frac{1}{2} \int_{0}^{T}\left\|\varphi_{t}\right\|^{2} \mathrm{~d} t\right] .
$$

The random variable $F\left(W+\varepsilon \int_{0}^{\cdot} \varphi_{s} \mathrm{~d} s\right)$ is a functional of the paths of a drift-perturbed multidimensional Brownian motion $W+\varepsilon \int_{0}^{*} \varphi_{s} \mathrm{~d} s$, where $\varepsilon$ is a small parameter and $\varphi$ is some adapted control process. Such a dependence typically arises because $F$ depends on a state variable $X^{(\varepsilon)}$ which is a perturbed process following

$$
\mathrm{d} X_{t}^{(\varepsilon)}=a_{t} \mathrm{~d} t+b_{t}\left(\mathrm{~d} W_{t}+\varepsilon \varphi_{t} \mathrm{~d} t\right)
$$

with $a, b$ adapted processes.

Date: June 29, 2018.

Many thanks to Giuseppe Benedetti for helpful comments, and to an Associate Editor and two anonymous referees for careful and insightful reading and suggestions which have improved the paper. 
The idea behind our approach is to view the drift $\varepsilon \varphi$ in (1.1) or (1.2) as a perturbation to Brownian paths on Wiener space. For $\varepsilon=0$ the optimal control is zero, and we suppose that the optimal control for small $\varepsilon$ will be a perturbation around zero. Ideas of Malliavin calculus arise in differentiating the objective function of the control problem with respect to $\varepsilon$ at $\varepsilon=0$. This uses Bismut's [6] approach to the stochastic calculus of variations, which exploits the Girsanov theorem to translate a drift adjustment into to a measure change, in order to perform differentiation on path space. Ultimately, this leads to an asymptotic expansion for the value function, valid for small $\varepsilon$. In the financial application to indifference pricing, $\varepsilon^{2}=\alpha$, so one obtains small risk aversion asymptotics. Similar ideas arise in entropy minimisation problems, which are the dual to pure investment problems with exponential utility, and we illustrate an example of this in a stochastic volatility model, in which the small parameter is $1-\rho^{2}, \rho$ being the correlation between the stock and its volatility. The power of this approach is that we can obtain results in non-Markovian models and for quite general path-dependent payoffs.

Entropy-weighted control problems have been treated using variational principles by Boué and Dupuis [7] (we thank a referee for pointing out this work to us), with a view to applications in large deviations theory. The result in [7] is a representation of the form

$$
-\log \mathbb{E}\left[\mathrm{e}^{-g(W)}\right]=\inf _{v} \mathbb{E}\left[\frac{1}{2} \int_{0}^{T}\left\|v_{s}\right\|^{2} \mathrm{~d} s+g\left(W+\int_{0} v_{s} \mathrm{~d} s\right)\right] .
$$

Bierkens and Kappen [5] develop the methods in [7] further and obtain formulae for the optimal control in (1.3) as a Malliavin derivative of the functional $g(W)$. These papers are in a similar spirit to ours in sharing a variational point of view. It would be interesting to see if future work could to relate the results in [7, 5] to ours.

Utility-based valuation techniques rarely lead to explicit solutions, and this motivates the interest in approximate solutions. The idea of using Malliavin calculus methods in asymptotic indifference pricing is due to Davis [10]. Davis used the approach in a two-dimensional constant parameter basis risk model, with a traded and non-traded asset following correlated geometric Brownian motions, and for a European claim depending only on the final value of the non-traded asset price. In this model, it turns out that partial differential equation (PDE) techniques, based on a Cole-Hopf transform applied to the the Hamilton-Jacobi-Bellman equation of the underlying utility maximisation problem (see Zariphopoulou [42, Henderson [18] and Monoyios [30]), lead to a closed form non-linear expectation representation for the indifference price. The asymptotic expansion obtained by Davis [10] can therefore also be obtained by applying a Taylor expansion to the non-linear expectation representation, as carried out in Monoyios [29, 32]. For this reason, perhaps, the technique developed by Davis has not been further exploited.

In higher-dimensional models, and in almost all models with random parameters, the aforementioned Cole-Hopf transform does not work. Indifference prices and their risk-aversion asymptotics have been analysed via other methods, notably by backward stochastic differential equation (BSDE) and bounded-mean-oscillation (BMO) martingale methods (Mania and Schweizer [28, Kallsen and Rheinländer [23]) for bounded claims. Monoyios [33] derived small risk aversion valuation and hedging results via PDE techniques, in a random parameter basis risk model generated by incomplete information on asset drifts. Delbaen et al [11] and Stricker [40] used arguments based on a Fenchel inequality to derive the zero risk aversion limit of the indifference price. Recently, Henderson and Liang [19] have used BSDE and PDE techniques to derive indifference price approximations, of a different nature to ours, in a multi-dimensional non-traded assets model.

The techniques in this paper are different. We resurrect the method suggested by Davis [10]. The first contribution is to show that this technique can be significantly generalised, to cover multi-dimensional Itô process markets, with no Markov structure required, and for claims which can be quite general functionals of the paths of the asset prices. In doing this we elucidate the precise relation with the Malliavin calculus. The second contribution is to derive a representation (Proposition 3.3) for the optimal control in problems of the form (1.1), using variational techniques on Wiener space. This is used in verifying the correct structure of our asymptotic expansion. 
The third contribution is to establish a dual stochastic control representation (Lemma 4.8) of the indifference price process in a semi-martingale model. This result seems to be the most compact representation possible. We apply the Malliavin asymptotic method to this control problem in an Itô process setting, and derive the general form of the small risk aversion asymptotic expansion of an exponential indifference price, recovering the well-known connection between small risk aversion exponential indifference valuation and quadratic risk minimisation. Examples are given of multi-asset basis risk models, and of stochastic correlation in basis risk. Finally, we show how the technique can be applied to identify the minimal entropy martingale measure (MEMM) $\mathbb{Q}^{0} \equiv \mathbb{Q}_{E}$ as a perturbation to the minimal martingale measure $\mathbb{Q}_{M}$ in a stochastic volatility model, when the stock and volatility are highly correlated.

Other types of asymptotic expansion for marginal utility-based prices, in terms of a small holding of claims, have been obtained by Kramkov and Sîrbu [25] and by Kallsen et al [22]. These works use utility functions defined on the positive half-line, in contrast to the exponential utility function used in this paper. In stochastic volatility models, Sircar and Zariphopoulou [39] obtain asymptotic expansions for exponential indifference prices using the fast mean-reversion property of the volatility process. This approach has been significantly exploited in many scenarios (see Sircar et al [14]), and is of a different nature to our approach.

Malliavin calculus has found application in other areas of mathematical finance, such as insider trading 20, to computation of sensitivity parameters [15], and to other forms of asymptotic expansion [3], involving sensitivity with respect to initial conditions, or with respect to parameters in asset price dynamics, or to parameters appearing in an expectation, as opposed to a control.

The rest of the paper is organised as follows. In Section 2 we prove a version of the Malliavin integration-by-parts formula on Wiener space (Lemma 2.2), giving a directional derivative of a Brownian functional. In Section 3 this is used to derive our asymptotic expansion (Theorem 3.1). We use variational methods to characterise the optimal control (Proposition 3.3) which helps us characterise the error term in the approximation. The interplay between directional derivatives on Wiener space, the Malliavin derivative, and perturbation analysis is exemplified in this section. In Section 4 we derive, in a locally bounded semi-martingale model, the dual stochastic control representation of the indifference price process (Lemma 4.8) that forms the basis of the financial control problems we are interested in. In Section 5 we apply the asymptotic analysis of indifference valuation in an Itô process setting. In Section 6 we give examples of approximate indifference valuation in some basis risk models, and we show how the MEMM can be identified as a perturbation to the minimal martingale measure in a stochastic volatility model.

\section{Directional Derivatives of Brownian functionals on Wiener space}

In this section we consider perturbations to Brownian paths, and the ensuing directional derivatives, on Wiener space. This is Bismut's [6] approach to the Malliavin calculus, and will be used in asymptotic analysis of control problems in the next section. In this approach, one deduces a certain invariance principle (see (2.17)) by using the Girsanov theorem to translate a drift perturbation to a Brownian motion into a change of probability measure. This approach is discussed in Section IV.41 of Rogers and Williams [36], and Appendix E of Karatzas and Shreve [24. Nualart [34] is a general treatise on Malliavin calculus.

The setting uses the canonical basis $\left(\Omega, \mathcal{F}, \mathbb{F}:=\left(\mathcal{F}_{t}\right)_{0 \leq t \leq T}, \mathbb{P}\right)$, on which we define an $m$ dimensional Brownian motion $W$. So, $\Omega=C_{0}\left([0, T] ; \mathbb{R}^{m}\right)$, the Banach space of continuous functions $\omega:[0, T] \rightarrow \mathbb{R}^{m}$ null at zero, equipped with the supremum norm $\|\omega(t)\|_{\infty}:=\sup _{t \in[0, T]}\|\omega(t)\|$, $\mathbb{P}$ is Wiener measure, and $\left(W_{t}(\omega):=\omega(t)\right)_{t \in[0, T]}$ is $m$-dimensional Brownian motion with natural filtration $\mathbb{F}$. The Malliavin calculus is conventionally introduced with reference to the Hilbert space $H=L^{2}\left([0, T], \mathcal{B}([0, T])\right.$, Leb; $\left.\mathbb{R}^{m}\right)$ (we write $H=L^{2}\left([0, T] ; \mathbb{R}^{m}\right)$ for brevity). An element $h \in H$ is a function $h:[0, T] \rightarrow \mathbb{R}^{m}$, with norm $\|h\|_{H}^{2}=\int_{0}^{T}\left\|h_{t}\right\|^{2} \mathrm{~d} t<\infty$. Then the Wiener 
integral $\mathbb{W}(h)$, defined by

$$
\mathbb{W}(h):=\sum_{i=1}^{m} \int_{0}^{T} h_{t}^{i} \mathrm{~d} W_{t}^{i} \equiv \int_{0}^{T} h_{t} \cdot \mathrm{d} W_{t} \equiv(h \cdot W)_{T},
$$

is an isonormal Gaussian process. That is, the linear isometry $\mathbb{W}: H \rightarrow L^{2}[(\Omega, \mathcal{F}, \mathbb{P}) ; \mathbb{R}]$ is such that $\mathbb{W}=(\mathbb{W}(h))_{h \in H}$ is a centred family of Gaussian random variables with $\mathbb{E}[\mathbb{W}(h)]=0$ and

$$
\mathbb{E}[\mathbb{W}(h) \mathbb{W}(g)]=\langle h, g\rangle_{H}=\int_{0}^{T} h_{t} \cdot g_{t} \mathrm{~d} t=\sum_{i=1}^{m} \int_{0}^{T} h_{t}^{i} g_{t}^{i} \mathrm{~d} t .
$$

For $\varphi \in H=L^{2}\left([0, T] ; \mathbb{R}^{m}\right)$, the Cameron-Martin subspace $\mathcal{C} \mathcal{M} \subset \Omega=C_{0}\left([0, T] ; \mathbb{R}^{m}\right)$ is composed of absolutely continuous functions $\Phi:[0, T] \rightarrow \mathbb{R}^{m}$ with square-integrable derivative $\varphi$. That is,

$$
\Phi_{t}:=\int_{0}^{t} \varphi_{s} \mathrm{~d} s, \quad \int_{0}^{t}\left\|\varphi_{s}\right\|^{2} \mathrm{~d} s<\infty, \quad 0 \leq t \leq T .
$$

One transports the Hilbert space structure of $H$ to $\mathcal{C M}$ by defining

$$
\langle\Phi, \Psi\rangle_{\mathcal{C M}}:=\langle\varphi, \psi\rangle_{H}=\int_{0}^{T} \varphi_{t} \cdot \psi_{t} \mathrm{~d} t, \quad \Psi:=\int_{0} \psi_{s} \mathrm{~d} s,
$$

so $\mathcal{C M}$ is isomorphic to $H$. If $F$ is Malliavin-differentiable, then there exists an $H$-valued random variable, so a measurable (but not necessarily adapted) process $\left(D_{t} F\right)_{t \in[0, T]}$, such that for $\Phi \in \mathcal{C M}$ we have the integration-by-parts formula

$$
\mathbb{E}\left[\langle D F, h\rangle_{H}\right]=\mathbb{E}[F \mathbb{W}(h)]
$$

or

$$
\mathbb{E}\left[\int_{0}^{T} D_{t} F \cdot \varphi_{t} \mathrm{~d} t\right]=\mathbb{E}\left[F \int_{0}^{T} \varphi_{t} \cdot \mathrm{d} W_{t}\right],
$$

and $\langle D F, h\rangle_{H}$ has properties of a directional derivative. This will be transparent in the Bismut approach to the Malliavin calculus, which we outline below.

2.1. The Bismut approach. Bismut [6] developed an alternative version of the stochastic calculus of variations, in which the left-hand-side of (2.1) is a directional derivative on Wiener space, and which allows for $\varphi$ to be a previsible process.

We have a square-integrable functional $F(W)$ of the Brownian paths $W$, that is, an $\mathcal{F}_{T^{-}}$ measurable map $F: \Omega \rightarrow \mathbb{R}$ satisfying

$$
\mathbb{E}\left[F^{2}(W)\right]<\infty .
$$

Let $\Phi \in C_{0}^{1}\left([0, T] ; \mathbb{R}^{m}\right) \subset \Omega$, with $\Phi:=\int_{0}^{*} \varphi_{s} \mathrm{~d} s$ for some previsible process $\varphi$. We are interested in defining a directional derivative of $F$ in the direction $\Phi$.

The first variation (or Gâteaux variation) $\delta F(W ; \Phi)$ of $F$ at $W \in \Omega$ in the direction $\Phi$ is the limit, if it exists, given by

$$
\delta F(W ; \Phi):=\lim _{\varepsilon \rightarrow 0} \frac{1}{\varepsilon}[F(W+\varepsilon \Phi)-F(W)]=\left.\frac{\mathrm{d}}{\mathrm{d} \varepsilon}[F(W+\varepsilon \Phi)]\right|_{\varepsilon=0} .
$$

(See Luenberger [27] (Chapter 7) or Wouk [41] (Chapter 12) for more on this and other notions of differentiation of non-linear maps in Banach spaces.) The first variation is homogeneous in the perturbation $\Phi: \delta F(W ; c \Phi)=c \delta F(W ; \Phi)$ for $c \in \mathbb{R}$. We are interested in the case when $F$ is such that the first variation is also linear in $\Phi$. To this end, we impose the following conditions on $F$, similar to those used in Appendix E of Karatzas and Shreve [24].

Assumption 2.1. $\quad$ (i) $F$ satisfies square-integrability condition (2.2).

(ii) There exists a non-negative Brownian functional $k$ satisfying $\mathbb{E}\left[k^{2}(W)\right]<\infty$ and a function $g:[0, \infty) \rightarrow[0, \infty)$ satisfying $\lim \sup _{\varepsilon \downarrow 0}(g(\varepsilon) / \varepsilon)<\infty$, such that for $W, \Phi \in \Omega$,

$$
|F(W+\Phi)-F(W)| \leq k(W) g\left(\|\Phi\|_{\infty}\right) .
$$


(iii) There exists a kernel $\partial F(\omega ; \cdot) \equiv \partial F(W ; \cdot): \Omega \rightarrow \mathbb{M}$, where $\mathbb{M}$ is the set of $m$-dimensional finite Borel measures on $[0, T]$, such that for each $\Phi \in C_{0}^{1}\left([0, T] ; \mathbb{R}^{m}\right) \subset \Omega$ we have

$$
\lim _{\varepsilon \rightarrow 0} \frac{1}{\varepsilon}[F(W+\varepsilon \Phi)-F(W)]=\int_{0}^{T} \Phi_{t} \cdot \partial F(W ; \mathrm{d} t), \quad \text { for almost all } W \in \Omega .
$$

Note, in particular, that (2.4) implies

$$
F(W+\varepsilon \Phi)=F(W)+\varepsilon \int_{0}^{T} \Phi_{t} \cdot \partial F(W ; \mathrm{d} t)+o\left(|\varepsilon|\|\Phi\|_{\infty}\right) .
$$

Using $\Phi=\int_{0}^{\cdot} \varphi_{s} \mathrm{~d} s$ on the right-hand-side of (2.4), we may integrate by parts to obtain the equivalent form

$$
\lim _{\varepsilon \rightarrow 0} \frac{1}{\varepsilon}\left[F\left(W+\varepsilon \int_{0}^{\cdot} \varphi_{s} \mathrm{~d} s\right)-F(W)\right]=\int_{0}^{T} \partial F(W ;(t, T]) \cdot \varphi_{t} \mathrm{~d} t .
$$

In particular, we then have the analogue of (2.5):

$$
F\left(W+\varepsilon \int_{0}^{\cdot} \varphi_{s} \mathrm{~d} s\right)=F(W)+\varepsilon \int_{0}^{T} \partial F(W ;(t, T]) \cdot \varphi_{t} \mathrm{~d} t+o\left(|\varepsilon|\|\varphi\|_{\infty}\right) .
$$

Rogers and Williams [36] (Section IV.41) make the observations that the condition (2.4) in Assumption 2.1 is automatically satisfied if $F$ is Fréchet differentiable with bounded derivative, and in that case $\partial F \equiv F^{\prime}$, where the Fréchet derivative $F^{\prime}(W ; \cdot)$ is a bounded linear functional on $\Omega$ (that is, a measure with finite total variation, an element of the dual space $\Omega^{\prime}$ ). But there are functionals where differentiability fails but (2.4) holds ([36, Section IV.41 has such examples).

Our particular interest will be in the functional $\mathbb{E}[F(W+\varepsilon \Phi)]$ and the associated variation

$$
\lim _{\varepsilon \rightarrow 0} \frac{1}{\varepsilon} \mathbb{E}[F(W+\varepsilon \Phi)-F(W)]=\left.\frac{\mathrm{d}}{\mathrm{d} \varepsilon} \mathbb{E}[F(W+\varepsilon \Phi)]\right|_{\varepsilon=0} .
$$

It turns out that one can make sense of this limit, resulting in a version of the integration-byparts formula (2.1) which holds regardless of whether $F$ is Malliavin differentiable. This is given in Lemma 2.2 further below.

2.1.1. The Clark formula. The classical result of Clark 8 relates the kernel $\partial F$ to the progressively measurable integrand $\psi$ (satisfying $\mathbb{E}\left[\int_{0}^{T}\left\|\psi_{t}\right\|^{2} \mathrm{~d} t\right]<\infty$ ) in the martingale representation of $F(W)$ :

$$
F(W)=\mathbb{E}[F(W)]+\int_{0}^{T} \psi_{t} \cdot \mathrm{d} W_{t} .
$$

The Clark formula gives $\psi$ as the predictable projection of the measurable (but not necessarily adapted) process $(\partial F(W ;(t, T]))_{0 \leq t \leq T}$. In other words,

$$
\psi_{t}=\mathbb{E}\left[\partial F(W ;(t, T]) \mid \mathcal{F}_{t}\right], \quad 0 \leq t \leq T .
$$

This result is proven in Appendix E of Karatzas and Shreve [24 and in Section IV.41 of Rogers and Williams [36], using similar methods to those that we shall employ in the proof of Lemma 2.2 below.

Lemma 2.2 (Directional derivative on Wiener space). Let $F \equiv F(W)$ be a functional of the Brownian paths $W$ on the Banach space $\Omega=C_{0}\left([0, T] ; \mathbb{R}^{m}\right)$ satisfying Assumption 2.1 . Let $\varphi$ be a bounded previsible process, with $\Phi \in C_{0}^{1}\left([0, T] ; \mathbb{R}^{m}\right) \subset \Omega$ defined by $\Phi:=\int_{0}^{\cdot} \varphi_{s} \mathrm{~d} s$. Then the map $\varepsilon \rightarrow \mathbb{E}[F(W+\varepsilon \Phi)]$ is differentiable, with derivative

$$
\left.\frac{\mathrm{d}}{\mathrm{d} \varepsilon} \mathbb{E}[F(W+\varepsilon \Phi)]\right|_{\varepsilon=0}=\mathbb{E}\left[F(W)(\varphi \cdot W)_{T}\right] .
$$

Moreover, if $\varphi=c \widetilde{\varphi}$ for some fixed $\widetilde{\varphi}$ and $c \in \mathbb{R}$, then

$$
\mathbb{E}\left[F(W+\varepsilon \Phi)-F(W)-\varepsilon F(W)(\varphi \cdot W)_{T}\right] \sim O\left(c^{2} \varepsilon^{2}\right) .
$$


A form of Lemma 2.2 appears in Davis [10] (his Lemma 3) in a one-dimensional set-up, with a functional dependent only on the final value of a diffusion. Fournié et al [15] have results of a similar nature in the context of perturbations arising from variations in the drift or diffusion coefficients of Markov SDEs (see, for instance, Proposition 3.1 in [15]).

To prove Lemma 2.2 we will need the following property of exponential martingales.

Lemma 2.3. For a bounded previsible process $\varphi$ and $\varepsilon \in \mathbb{R}$, define the exponential martingale

$$
M_{t}^{(\varepsilon)}:=\mathcal{E}(-\varepsilon \varphi \cdot W)_{t}:=\exp \left(-\varepsilon \int_{0}^{t} \varphi_{s} \cdot \mathrm{d} W_{s}-\frac{1}{2} \varepsilon^{2} \int_{0}^{t}\left\|\varphi_{s}\right\|^{2} \mathrm{~d} s\right), \quad 0 \leq t \leq T .
$$

Then we have

$$
\lim _{\varepsilon \rightarrow 0} \mathbb{E}\left[\int_{0}^{t}\left(1-M_{s}^{(\varepsilon)}\right)^{2} \mathrm{~d} s\right]=0, \quad 0 \leq t \leq T
$$

and

$$
\frac{1}{\varepsilon}\left(1-M_{t}^{(\varepsilon)}\right) \rightarrow(\varphi \cdot W)_{t}, \quad \text { in } L^{2} \text {, as } \varepsilon \rightarrow 0, \text { for every } t \in[0, T] .
$$

Proof. Since $\varphi$ is bounded, Novikov's criterion is satisfied and $M^{(\varepsilon)}$ is a martingale. Using the representation

$$
M_{t}^{(\varepsilon)}=1-\varepsilon \int_{0}^{t} M_{s}^{(\varepsilon)} \varphi_{s} \cdot \mathrm{d} W_{s}, \quad 0 \leq t \leq T,
$$

the stochastic integral is a martingale and we have

$$
\mathbb{E}\left[\int_{0}^{t}\left(M_{s}^{(\varepsilon)}\right)^{2}\left\|\varphi_{s}\right\|^{2} \mathrm{~d} s\right]<\infty, \quad 0 \leq t \leq T
$$

Using (2.15) along with the Itô isometry, we have, for any $t \in[0, T]$,

$$
\mathbb{E}\left[\int_{0}^{t}\left(1-M_{s}^{(\varepsilon)}\right)^{2} \mathrm{~d} s\right]=\varepsilon^{2} \mathbb{E}\left[\int_{0}^{t} \int_{0}^{s}\left(M_{u}^{(\varepsilon)}\right)^{2}\left\|\varphi_{u}\right\|^{2} \mathrm{~d} u \mathrm{~d} s\right] .
$$

By (2.16), the expectation on the right-hand-side is finite for any value of $\varepsilon$. Hence, letting $\varepsilon \rightarrow 0$ we obtain (2.13).

Using (2.15) and the Itô isometry once again, we compute, for any $t \in[0, T]$,

$$
\mathbb{E}\left[\left(\frac{1}{\varepsilon}\left(1-M_{t}^{(\varepsilon)}\right)-(\varphi \cdot W)_{t}\right)^{2}\right]=\mathbb{E}\left[\int_{0}^{t}\left(1-M_{s}^{(\varepsilon)}\right)^{2}\left\|\varphi_{s}\right\|^{2} \mathrm{~d} s\right],
$$

which, using (2.13) and the fact that $\varphi$ is bounded, converges to zero as $\varepsilon \rightarrow 0$, and this gives (2.14).

Proof of Lemma 2.2. We use a version of arguments found in some proofs of the Clark representation formula (see, for instance, Appendix E of Karatzas and Shreve 24] or the proof of Theorem IV.41.9 in Rogers and Williams [36]).

For $\varepsilon \in \mathbb{R}$ and $\varphi$ previsible and bounded, define the probability measure $\mathbb{P}^{(\varepsilon)}$ by

$$
\frac{\mathrm{d} \mathbb{P}^{(\varepsilon)}}{\mathrm{d} \mathbb{P}}=M_{T}^{(\varepsilon)}
$$

where $M^{(\varepsilon)}$ is the exponential martingale defined in (2.12). By the Girsanov Theorem, $W+\varepsilon \Phi$ is Brownian motion under $\mathbb{P}^{(\varepsilon)}$, so that with $\mathbb{E}^{(\varepsilon)}$ denoting expectation under $\mathbb{P}^{(\varepsilon)}$,

$$
\mathbb{E}[F(W)]=\mathbb{E}^{(\varepsilon)}[F(W+\varepsilon \Phi)]=\mathbb{E}\left[M_{T}^{(\varepsilon)} F(W+\varepsilon \Phi)\right] .
$$

This invariance principle underlies Bismut's approach to the Malliavin calculus. 
Re-write (2.17) as

$$
\mathbb{E}\left[\frac{F(W+\varepsilon \Phi)-F(W)}{\varepsilon}\right]=\mathbb{E}\left[\frac{1-M_{T}^{(\varepsilon)}}{\varepsilon} F(W)\right]+\mathbb{E}\left[\frac{F(W+\varepsilon \Phi)-F(W)}{\varepsilon}\left(1-M_{T}^{(\varepsilon)}\right)\right]
$$

We differentiate $\mathbb{E}[F(W+\varepsilon \Phi)]$ with respect to $\varepsilon$ at $\varepsilon=0$ by considering what happens when we let $\varepsilon \rightarrow 0$ in (2.18). To this end, subtract $\mathbb{E}\left[F(W)(\varphi \cdot W)_{T}\right]$ from both sides, to compute

$$
\begin{aligned}
& \mathbb{E}\left[\frac{1}{\varepsilon}(F(W+\varepsilon \Phi)-F(W))-F(W)(\varphi \cdot W)_{T}\right] \\
= & \mathbb{E}\left[\left(\frac{1-M_{T}^{(\varepsilon)}}{\varepsilon}-(\varphi \cdot W)_{T}\right) F(W)\right]+\mathbb{E}\left[\frac{F(W+\varepsilon \Phi)-F(W)}{\varepsilon}\left(1-M_{T}^{(\varepsilon)}\right)\right] .
\end{aligned}
$$

Now take the limit $\varepsilon \rightarrow 0$ in (2.19). Using conditions (i) and (ii) in Assumption (2.1), the dominated convergence theorem and the Cauchy-Schwarz inequality, the last term on the righthand-side is bounded by

$$
\mathbb{E}\left[k(W)\left(g\left(|\varepsilon|\|\Phi\|_{\infty}\right) /|\varepsilon|\right)\left|1-M_{T}^{(\varepsilon)}\right|\right] \leq K\left(\mathbb{E}\left[\left(1-M_{T}^{(\varepsilon)}\right)^{2}\right]\right)^{1 / 2}, \quad \text { for some constant } K,
$$

which converges to zero as $\varepsilon \rightarrow 0$, because of (2.13).

Next consider the first term on the right-hand-side of (2.19). Using the square-integrability of $F$ and the Cauchy-Schwarz inequality, we have

$$
\left(\mathbb{E}\left[\left(\frac{1-M_{T}^{(\varepsilon)}}{\varepsilon}-(\varphi \cdot W)_{T}\right) F(W)\right]\right)^{2} \leq K \mathbb{E}\left[\left(\frac{1-M_{T}^{(\varepsilon)}}{\varepsilon}-(\varphi \cdot W)_{T}\right)^{2}\right]
$$

for some constant $K$. This converges to zero as $\varepsilon \rightarrow 0$, on using (2.14). Thus the right-hand-side, and hence the left-hand-side, of (2.19) converges to zero as $\varepsilon \rightarrow 0$ and this establishes (2.10), the first part of the lemma.

To establish (2.11), we apply the same arguments to (2.19) multiplied by $\varepsilon$. We have

$$
\begin{aligned}
& \mathbb{E}\left[F(W+\varepsilon \Phi)-F(W)-\varepsilon F(W)(\varphi \cdot W)_{T}\right] \\
= & \mathbb{E}\left[\left(1-M_{T}^{(\varepsilon)}-\varepsilon(\varphi \cdot W)_{T}\right) F(W)\right]+\mathbb{E}\left[(F(W+\varepsilon \Phi)-F(W))\left(1-M_{T}^{(\varepsilon)}\right)\right] .
\end{aligned}
$$

We examine how each of the terms on the right-hand-side scale for small $\varepsilon$ and $\varphi=c \widetilde{\varphi}$. Using the representation (2.15), the second term satisfies

$$
\mathbb{E}\left[(F(W+\varepsilon \Phi)-F(W))\left(1-M_{T}^{(\varepsilon)}\right)\right] \leq \varepsilon \mathbb{E}\left[k(W) g\left(|\varepsilon|\|\Phi\|_{\infty}\right)\left|\int_{0}^{T} M_{t}^{(\varepsilon)} \varphi_{t} \cdot \mathrm{d} W_{t}\right|\right],
$$

and so for $\varphi=c \widetilde{\varphi}$ this term is of $O\left(c^{2} \varepsilon^{2}\right)$, on invoking the properties of $g(\cdot)$ in Assumption 2.1 (ii). For the first term, using the representation (2.15) for $\varphi=c \widetilde{\varphi}$, we have

$$
\mathbb{E}\left[\left(1-M_{T}^{(\varepsilon)}-\varepsilon(\varphi \cdot W)_{T}\right) F(W)\right]=c^{2} \varepsilon^{2} \mathbb{E}\left[F(W) \int_{0}^{T} \frac{1}{c \varepsilon}\left(M_{t}^{(\varepsilon)}-1\right) \widetilde{\varphi}_{t} \cdot \mathrm{d} W_{t}\right],
$$

which is is of $O\left(c^{2} \varepsilon^{2}\right)$, on using (2.14). Hence (2.11) is established.

Remark 2.4. The boundedness condition on $\varphi$ in Lemma 2.2 can be relaxed. A Novikov condition on $\varepsilon \varphi$ would suffice, so that the stochastic exponential $M^{(\varepsilon)}$ in (2.12) is a martingale. This remark also pertains to Lemma 2.3 . 
2.2. Relation with the Malliavin derivative. We can connect the Malliavin derivative of $F$ (when this exists) to the kernel $\partial F$ in the condition (2.4) and to the directional derivative in Lemma 2.2.

First, note that the right-hand-side of (2.10) has the same structure as the right-hand-side of (2.1), so Lemma 2.2 is a version of the integration-by-parts formula, generalised to Bismut's set-up, when $\Phi=\int_{0}^{*} \varphi_{s} \mathrm{~d} s$ need not be restricted to elements of $\mathcal{C M}$.

Now, with $\Phi=\int_{0}^{0} \varphi_{s} \mathrm{~d} s$, take the limit $\varepsilon \rightarrow 0$ in (2.18), using the conditions in Assumption 2.1 and the Dominated Convergence Theorem (a similar procedure is used in [24, 36] in proving the Clark representation formula), to obtain

$$
\mathbb{E}\left[\int_{0}^{T} \Phi_{t} \cdot \partial F(W ; \mathrm{d} t)\right]=\mathbb{E}\left[F(W) \int_{0}^{T} \varphi_{t} \cdot \mathrm{d} W_{t}\right]
$$

Using $\Phi=\int_{0}^{\cdot} \varphi_{s} \mathrm{~d} s$ and integrating by parts on the left-hand-side as was done to obtain (2.6), we convert (2.20) to the equivalent form

$$
\mathbb{E}\left[\int_{0}^{T} \partial F(W ;(t, T]) \cdot \varphi_{t} \mathrm{~d} t\right]=\mathbb{E}\left[F(W) \int_{0}^{T} \varphi_{t} \cdot \mathrm{d} W_{t}\right] .
$$

Comparing with (2.10), we see that the left-hand-side of (2.21) is just another way to write the directional derivative in Lemma 2.2. Note that if we use the martingale representation (2.8) of $F$ on the right-hand-side of (2.21) we obtain the Clark formula (2.9).

In the case that $\Phi \equiv \int_{0}^{\infty} \varphi_{s} \mathrm{~d} s$ is an element of the Cameron-Martin space $\mathcal{C} \mathcal{M}$, and for Malliavin-differentiable $F$, the right-hand-side of (2.10) or (2.21) is also the right-hand-side of the integration-by-parts formula (2.1), so in this case the kernel $\partial F$ is related to the Malliavin derivative according to

$$
\partial F(W ;(t, T])=D_{t} F(W), \quad 0 \leq t \leq T,
$$

and (2.21) is the integration-by-parts formula. So when $F$ is Malliavin-differentiable and $\Phi \in$ $\mathcal{C M} \subset \Omega$, the directional derivative in (2.10) is also given by $\mathbb{E}\left[\int_{0}^{T} D_{t} F \cdot \varphi_{t} \mathrm{~d} t\right]$. But Lemma 2.2 is valid when $F$ is not necessarily Malliavin-differentiable and for previsible $\varphi$, with $\Phi \equiv \int_{0}^{\cdot} \varphi_{s} \mathrm{~d} s$ not necessarily in $\mathcal{C M}$.

\section{Malliavin asymptotics of a CONTROL PRoblem}

In this section we describe a control problem and analyse it via variational principles and Bismut-Malliavin asymptotics. How this type of problem arises in a financial model will be described in subsequent sections.

We have a canonical basis $\left(\Omega, \mathcal{F}, \mathbb{F}=\left(\mathcal{F}_{t}\right)_{0 \leq t \leq T}, \mathbb{P}\right)$, on which is defined an $m$-dimensional Brownian motion $W$. A square-integrable random variable $F$ is a functional of the paths of the perturbed Brownian motion $W+\varepsilon \int_{0}^{\cdot} \varphi_{s} \mathrm{~d} s$, where $\varepsilon \in \mathbb{R}$ is a small parameter and $\varphi$ is a control process satisfying $\int_{0}^{T}\left\|\varphi_{t}\right\|^{2} \mathrm{~d} t<\infty$, and such that $\mathcal{E}(|\varepsilon| \varphi \cdot W$ ) is a martingale. (A Novikov condition $\mathbb{E}\left[\exp \left(\frac{1}{2} \varepsilon^{2} \int_{0}^{T}\left\|\varphi_{t}\right\|^{2} \mathrm{~d} t\right)\right]<\infty$ would suffice). Denote by $\mathcal{A}$ the set of such controls.

The control problem we are interested in is to maximise an objective functional $G(\varphi)$, defined by

$$
G(\varphi):=\mathbb{E}\left[F\left(W+\varepsilon \int_{0}^{.} \varphi_{s} \mathrm{~d} s\right)-\frac{1}{2} \int_{0}^{T}\left\|\varphi_{t}\right\|^{2} \mathrm{~d} t\right] .
$$

The value function is

$$
p:=G\left(\varphi^{*}\right)=\sup _{\varphi \in \mathcal{A}} G(\varphi)
$$

for some optimal control $\varphi^{*}$. 
As we shall see in Section [5, in finance this type of control problem typically arises because $F$ is a functional of the Brownian paths through dependence on some perturbed state variable $X \equiv X^{(\varepsilon)} \in \mathbb{R}^{m}$, following an Itô process

$$
\mathrm{d} X_{t}^{(\varepsilon)}=a_{t} \mathrm{~d} t+b_{t}\left(\mathrm{~d} W_{t}+\varepsilon \varphi_{t} \mathrm{~d} t\right)
$$

for some $m$-dimensional adapted process $a$ satisfying $\int_{0}^{T}\left\|a_{t}\right\| \mathrm{d} t<\infty$ and $m \times m$ adapted matrix process $b$ satisfying $\int_{0}^{T}\left\|a_{t}\right\| \mathrm{d} t<\infty$ and $\int_{0}^{T} b_{t} b_{t}^{\top} \mathrm{d} t<\infty$. In this section we shall not require a state process $X^{(\varepsilon)}$.

The idea behind the asymptotic expansion is to treat $\varepsilon \varphi$ as a perturbation to the Brownian paths. We suppose that, for small $\varepsilon$, the optimal control $\varphi^{*}$ will be small. We expand the objective functional in (3.1) about $\varepsilon=0$ using Lemma 2.2. Naturally, for $\varepsilon=0$ the functional $F\left(W+\varepsilon \int_{0}^{*} \varphi_{s} \mathrm{~d} s\right)$ loses all dependence on the control $\varphi$, so in this case optimal control is zero, and the leading order term will be $\mathbb{E}[F(W)]$. Ultimately, this leads to the main result below, a perturbative solution to the control problem (3.2).

Theorem 3.1. On the canonical basis $\left(\Omega, \mathcal{F},\left(\mathcal{F}_{t}\right)_{t \in[0, T]}, \mathbb{P}\right)$, define an m-dimensional Brownian motion $W$. Let $\Phi:=\int_{0}^{\cdot} \varphi_{s} \mathrm{~d} s \in \Omega$ be such that $\int_{0}^{T}\left\|\varphi_{t}\right\|^{2} \mathrm{~d} t<\infty$ and $\mathcal{E}(|\varepsilon| \varphi \cdot W)$ is a martingale, with $\varepsilon \in \mathbb{R}$ a small parameter. Denote the set of such $\varphi$ by $\mathcal{A}$. Let $F\left(W+\varepsilon \int_{0}^{\cdot} \varphi_{s} \mathrm{~d} s\right)$ be a functional of the paths of the perturbed Brownian motion $W+\varepsilon \int_{0}^{*} \varphi_{s} \mathrm{~d} s$, and satisfying Assumption 2.1. Then the control problem with value function (3.2) has asymptotic value given by

$$
p=\mathbb{E}[F(W)]+\frac{1}{2} \varepsilon^{2} \mathbb{E}\left[\int_{0}^{T}\left\|\psi_{t}\right\|^{2} \mathrm{~d} t\right]+O\left(\varepsilon^{4}\right),
$$

where $\psi$ is the integrand in the martingale representation (2.8) of $F(W)$.

Remark 3.2 (Heuristics). Before proving the theorem, we outline the ideas underlying the proof in a simple setting where ordinary calculus can replace variational calculus on Wiener space.

Consider maximising, over a scalar variable $\varphi$, a smooth function $J(\varepsilon, \varphi)$ given by

$$
J(\varepsilon, \varphi):=f(x+\varepsilon \varphi)-\frac{1}{2} \varphi^{2},
$$

for some smooth function $f$, and with $\varepsilon$ a small parameter. The optimiser of this problem satisfies

$$
\varphi^{*}=\varepsilon f^{\prime}\left(x+\varepsilon \varphi^{*}\right),
$$

so is of course zero for $\varepsilon=0$. If we seek a power series approximation for $\varphi^{*}$, writing $\varphi^{*}=$ $\sum_{k=1}^{\infty} \varepsilon^{k} \varphi^{(k)}$ for some coefficients $\varphi^{(k)}$, then using this in (3.6) along with a Taylor expansion of $f^{\prime}(x+\varepsilon \varphi)$ gives

$$
\varphi^{*}=\varepsilon f^{\prime}(x)\left(1+\varepsilon^{2} f^{\prime \prime}(x)\right)+O\left(\varepsilon^{5}\right) .
$$

In particular, the first two terms in $\varphi^{*}$ are linear and cubic in $\varepsilon$. With the given structure of the objective function in (3.5), this implies that the maximum has asymptotic expansion given by

$$
J\left(\varepsilon, \varphi^{*}\right)=f(x)+\frac{1}{2} \varepsilon^{2}\left(f^{\prime}(x)\right)^{2}+O\left(\varepsilon^{4}\right) .
$$

But this is the same value as is obtained by maximising the linear-in- $\varepsilon$ approximation to $J(\varepsilon, \varphi)$ :

$$
J(\varepsilon, \varphi)=f(x)+\varepsilon \varphi f^{\prime}(x)-\frac{1}{2} \varphi^{2}+O\left(\varepsilon^{2} \varphi^{2}\right),
$$

which is maximised by $\widehat{\varphi}=\varepsilon f^{\prime}(x)$, yielding

$$
J(\varepsilon, \widehat{\varphi})=f(x)+\frac{1}{2} \varepsilon^{2}\left(f^{\prime}(x)\right)^{2}+O\left(\varepsilon^{4}\right),
$$

so that $J\left(\varepsilon, \varphi^{*}\right)=J(\varepsilon, \widehat{\varphi})$ to order $\varepsilon^{2}$, with the remainder being of order $\varepsilon^{4}$ in both cases.

We shall see that a similar structure underlies the proof of Theorem 3.1 which we give further below, after some preparation. 
The following result is the analogue of (3.6) for differentiation (in a variational sense) of the the objective functional of the control problem with respect to the control $\varphi$. We will use this later in establishing the asymptotic expansion of Theorem 3.1 .

Proposition 3.3. Assume the same setting as in Theorem 3.1. The optimal control $\varphi^{*}$ for the problem with value function (3.2) satisfies

$$
\varphi_{t}^{*}=\varepsilon \mathbb{E}\left[\partial F\left(W+\varepsilon \int_{0}^{\cdot} \varphi_{s}^{*} \mathrm{~d} s ;(t, T]\right) \mid \mathcal{F}_{t}\right], \quad 0 \leq t \leq T,
$$

where $\partial F\left(W+\varepsilon \int_{0}^{*} \varphi_{s}^{*} \mathrm{~d} s ; \cdot\right)$ is the kernel in 2.4), evaluated at $W+\varepsilon \int_{0}^{\cdot} \varphi_{s}^{*} \mathrm{~d} s \in \Omega$.

Proof. Recall the conditions (2.3) and (2.4) in Assumption 2.1. We shall use these to differentiate, in a variational manner akin to our development of Lemma 2.2, the objective functional (3.1) of the control problem with respect to the control $\varphi$.

Consider varying $\varphi$ in (3.1). To this end, for $\gamma \in \mathbb{R}$ a small parameter and $\Xi=\int_{0}^{\cdot} \xi_{s} \mathrm{~d} s \in \Omega$, consider the variation

$$
\delta G(\varphi ; \xi):=\lim _{\gamma \rightarrow 0} \frac{1}{\gamma}[G(\varphi+\gamma \xi)-G(\varphi)] .
$$

Using (2.3) and (2.4) applied at $W+\epsilon \int_{0}^{*} \varphi_{s} \mathrm{~d} s$ along with the Dominated Convergence Theorem, we obtain

$$
\delta G(\varphi ; \xi)=\mathbb{E}\left[\int_{0}^{T} \varepsilon \Xi_{t} \cdot \partial F\left(W+\varepsilon \int_{0}^{.} \varphi_{s} \mathrm{~d} s ; \mathrm{d} t\right)-\int_{0}^{T} \varphi_{t} \cdot \xi_{t} \mathrm{~d} t\right] .
$$

Using $\Xi=\int_{0}^{\cdot} \xi_{s} \mathrm{~d} s$ and integrating by parts in the first term on the right-hand-side converts this to

$$
\delta G(\varphi ; \xi)=\mathbb{E}\left[\int_{0}^{T}\left(\varepsilon \partial F\left(W+\varepsilon \int_{0}^{\cdot} \varphi_{s} \mathrm{~d} s ;(t, T]\right)-\varphi_{t}\right) \cdot \xi_{t} \mathrm{~d} t\right] .
$$

The first order condition for the optimal control, $\delta G\left(\varphi^{*} ; \xi\right)=0$, gives that

$$
\mathbb{E}\left[\int_{0}^{T} \varepsilon \partial F\left(W+\varepsilon \int_{0}^{\cdot} \varphi_{s}^{*} \mathrm{~d} s ;(t, T]\right) \cdot \xi_{t} \mathrm{~d} t\right]=\mathbb{E}\left[\int_{0}^{T} \varphi_{t}^{*} \cdot \xi_{t} \mathrm{~d} t\right]
$$

must hold for every adapted process $\xi$, so (3.7) follows. Note that this is the analogue of (3.6) when performing variational differentiation on Wiener space.

Remark 3.4. If $F$ were Fréchet-differentiable (respectively, Malliavin-differentiable with controls $\varphi$ such that $\left.\int_{0}^{\cdot} \varphi_{s} \mathrm{~d} s \in \mathcal{C} \mathcal{M}\right)$ then the optimiser would be given by

$\varphi_{t}^{*}=\varepsilon \mathbb{E}\left[F^{\prime}\left(W+\varepsilon \int_{0}^{*} \varphi_{s}^{*} \mathrm{~d} s ;(t, T]\right) \mid \mathcal{F}_{t}\right]$ (respectively, $\left.\varphi_{t}^{*}=\varepsilon \mathbb{E}\left[D_{t} F\left(W+\varepsilon \int_{0}^{*} \varphi_{s}^{*} \mathrm{~d} s\right)\right) \mid \mathcal{F}_{t}\right]$.

Proof of Theorem 3.1. There are two parts to the proof. First, following the method of Davis [10], we use Lemma 2.2 to approximate $G(\varphi)$ for small $\varepsilon$, and maximise the approximation with respect to $\varphi$. We then show that if one were able to solve the problem exactly, and then approximate the value function $G\left(\varphi^{*}\right)$ for small $\varepsilon$, the same result would ensue. This will use variational arguments and Proposition 3.3 .

Using Lemma 2.2 and the martingale representation (2.8) of $F(W)$, the objective functional $G(\varphi)$ in (3.1) is approximated as

$$
G(\varphi)=\mathbb{E}\left[F(W)+\int_{0}^{T}\left(\varepsilon \psi_{t} \cdot \varphi_{t}-\frac{1}{2}\left\|\varphi_{t}\right\|^{2}\right) \mathrm{d} t\right]+o\left(|\varepsilon|\|\varphi\|_{\infty}\right) .
$$

This is maximised over $\varphi$ by choosing $\varphi=\widehat{\varphi}:=\varepsilon \psi$, to give

$$
G(\widehat{\varphi})=\mathbb{E}[F(W)]+\frac{1}{2} \varepsilon^{2} \mathbb{E}\left[\int_{0}^{T}\left\|\psi_{t}\right\|^{2} \mathrm{~d} t\right]+O\left(\varepsilon^{4}\right),
$$

with the remainder term of $O\left(\varepsilon^{4}\right)$ due to (2.11). Thus, (3.4) is indeed obtained by optimising the approximation to $G(\varphi)$. 
For the second part of the proof: using (2.7) along with (2.3) and the Dominated Convergence Theorem, we write the value function $G\left(\varphi^{*}\right)$ as

$$
G\left(\varphi^{*}\right)=\mathbb{E}\left[F(W)+\varepsilon \int_{0}^{T} \partial F(W ;(t, T]) \cdot \varphi_{t}^{*} \mathrm{~d} t-\frac{1}{2} \int_{0}^{T}\left\|\varphi_{t}^{*}\right\|^{2} \mathrm{~d} t\right]+o\left(|\varepsilon|\left\|\varphi^{*}\right\|_{\infty}\right) .
$$

From (3.7), it is evident that (under the mild condition that $\partial F$ possesses a well-defined first variation)

$$
\varphi_{t}^{*}=\varepsilon \mathbb{E}\left[\partial F(W ;(t, T]) \mid \mathcal{F}_{t}\right]+o\left(|\varepsilon|\left\|\varphi^{*}\right\|_{\infty}\right)=\epsilon \psi_{t}+o\left(|\varepsilon|\left\|\varphi^{*}\right\|_{\infty}\right),
$$

the last equality following from the Clark formula (2.9). Observe that, to first order in $\varepsilon, \varphi^{*}=\widehat{\varphi}$.

We now show what would happen if we were to impose a perturbative structure on the optimal control, that is, if we were to write

$$
\varphi_{t}^{*}=\varepsilon \psi_{t}+\varepsilon^{2} \varphi_{t}^{(2)}+\varepsilon^{3} \varphi_{t}^{(3)}+O\left(\varepsilon^{4}\right),
$$

for some coefficients $\varphi^{(2)}, \varphi^{(3)}$. Supposing such an expansion were possible, and using this in (3.9), we would have

$$
\varepsilon \psi_{t}+\varepsilon^{2} \varphi_{t}^{(2)}+\varepsilon^{3} \varphi_{t}^{(3)}+O\left(\varepsilon^{4}\right)=\varepsilon \psi_{t}+o\left(\varepsilon^{2}\|\psi\|_{\infty}\right) .
$$

This would imply, in particular, that $\varphi^{(2)}=0$, and then (3.10) converts to

$$
\varepsilon \varphi_{t}^{*}=\varepsilon^{2} \psi_{t}+O\left(\varepsilon^{4}\right) \text {. }
$$

Using this in (3.8) we obtain

$$
G\left(\varphi^{*}\right)=\mathbb{E}\left[F(W)+\varepsilon^{2} \int_{0}^{T} \partial F(W ;(t, T]) \cdot \psi_{t} \mathrm{~d} t-\frac{1}{2} \varepsilon^{2} \int_{0}^{T}\left\|\psi_{t}\right\|^{2} \mathrm{~d} t\right]+O\left(\varepsilon^{4}\right) .
$$

One can use iterated expectations and (2.9) to convert this to the statement (3.4) of the theorem.

Remark 3.5. For $F$ sufficiently Fréchet differentiable (respectively Malliavin differentiable with $\left.\int_{0}^{\cdot} \varphi_{s} \mathrm{~d} s \in \mathcal{C} \mathcal{M}\right)$, the proof of the asymptotic expansion and the quantification of the error term would be more straightforward, using a Taylor expansion of $F\left(W+\varepsilon \int_{0}^{\cdot} \varphi_{s} \mathrm{~d} s\right)$ and of the optimal control $\varphi_{t}^{*}=\mathbb{E}\left[F^{\prime}\left(W+\varepsilon \int_{0}^{\cdot} \varphi_{s}^{*} \mathrm{~d} s ;(t, T]\right) \mid \mathcal{F}_{t}\right]$ (respectively, $\left.\varphi_{t}^{*}=\mathbb{E}\left[D_{t} F\left(W+\varepsilon \int_{0}^{\cdot} \varphi_{s}^{*} \mathrm{~d} s ;(t, T]\right) \mid \mathcal{F}_{t}\right]\right)$.

\section{Dynamic dual Representations of indifference price processes}

In this section we derive a dynamic dual stochastic control representation for the exponential indifference price process of a European claim in a locally bounded semi-martingale market. This will form the basis for our asymptotic expansion of the indifference price. Our representation is a slight deviation from the usual way of expressing the indifference price in terms of relative entropy. Although the material in this section is mainly classical, we want a unified treatment that gives dynamic results for unbounded claims, and this is not readily available in one compact account.

Our approach is to begin with the seminal representation of Grandits and Rheinländer [17] and Kabanov and Stricker 21] for an entropy-minimising measure, to establish a dynamic version of this (Corollary 4.4), and to use this to establish a dynamic version (Theorem 4.5) of the duality result of Delbaen et al [11. This result has been obtained for a bounded claim by Mania and Schweizer [28]. We carry out this program for a claim satisfying exponential moment conditions akin to those in Becherer [2. Once we establish duality for the investment problem with random endowment, we obtain a dynamic version of the classical dual indifference price representation (Corollary 4.6). Then we derive a dynamic result on the entropic distance between measures (Proposition 4.7) using the results of [17, 21] once more, and this allows us to convert the classical indifference price representation to our required representation in Lemma 4.8.

The setting is a probability space $(\Omega, \mathcal{F}, \mathbb{P})$ equipped with a filtration $\mathbb{F}=\left(\mathcal{F}_{t}\right)_{0 \leq t \leq T}$ satisfying the usual conditions of right-continuity and completeness, where $T \in(0, \infty)$ is a fixed time horizon. We assume that $\mathcal{F}_{0}$ is trivial and that $\mathcal{F}=\mathcal{F}_{T}$. The discounted prices of $d$ stocks are modelled by 
a positive locally bounded semi-martingale $S$. Since we work with discounted assets, our formulae are unencumbered by any interest rate adjustments. The class $\mathbf{M}$ of equivalent local martingale measures (ELMMs) $\mathbb{Q}$ is of course defined by

$$
\mathbf{M}:=\{\mathbb{Q} \sim \mathbb{P} \mid S \text { is a } \mathbb{Q} \text {-local martingale }\},
$$

and is assumed non-empty. This assumption is a classical one, consistent with the absence of arbitrage opportunities, in accordance with Delbaen and Schachermayer 12 .

Denote by $Z^{\mathbb{Q}}$ the density process with respect to $\mathbb{P}$ of any $\mathbb{Q} \in \mathbf{M}$. We write $Z^{\mathbb{Q}, \mathbb{M}}$ for the density process of $\mathbb{Q} \in \mathbf{M}$ with respect to any measure $\mathbb{M}$ other than the physical measure $\mathbb{P}, \mathbb{E}^{\mathbb{M}}$ for expectation with respect to $\mathbb{M}$, and $\mathbb{E}$ for $\mathbb{E}^{\mathbb{P}}$.

For $0 \leq t \leq T$, we write $Z_{t, T}^{\mathbb{Q}}:=Z_{T}^{\mathbb{Q}} / Z_{t}^{\mathbb{Q}}$, with a similar convention for any positive process. The conditional relative entropy between $\mathbb{Q} \in \mathbf{M}$ and $\mathbb{P}$ is the process defined by

$$
I_{t}(\mathbb{Q} \mid \mathbb{P}):=\mathbb{E}^{\mathbb{Q}}\left[\log Z_{t, T}^{\mathbb{Q}} \mid \mathcal{F}_{t}\right], \quad 0 \leq t \leq T,
$$

provided this is almost surely finite. Define the subset of $\mathbf{M}$ given by

$$
\mathbf{M}_{f}:=\left\{\mathbb{Q} \in \mathbf{M} \mid I_{0}(\mathbb{Q} \mid \mathbb{P})<\infty\right\}
$$

and we assume throughout that this set of ELMMs with finite relative entropy is non-empty: $\mathbf{M}_{f} \neq \emptyset$. By Theorem 2.1 of Frittelli [16], this implies that there exists a unique $\mathbb{Q}^{0} \in \mathbf{M}_{f}$, the minimal entropy martingale measure $(\mathrm{MEMM})$, that minimises $I_{0}(\mathbb{Q} \mid \mathbb{P})$ over all $\mathbb{Q} \in \mathbf{M}_{f}$. It is well-known (for example, Proposition 4.1 of Kabanov and Stricker [21]) that the density process $Z^{\mathbb{Q}^{0}}$ also minimises the conditional relative entropy process $I(\mathbb{Q} \mid \mathbb{P})$ between $\mathbb{Q} \in \mathbf{M}_{f}$ and $\mathbb{P}$.

The density process of one martingale measure with respect to another is simply the ratio of their density processes with respect to $\mathbb{P}$, as shown in the following lemma.

Lemma 4.1. Let $\mathbb{Q}_{1}, \mathbb{Q}_{2} \in \mathbf{M}_{f}$ have density processes $Z^{\mathbb{Q}_{1}}, Z^{\mathbb{Q}_{2}}$ with respect to $\mathbb{P}$. Then the density process of $\mathbb{Q}_{1}$ with respect to $\mathbb{Q}_{2}$ is $Z^{\mathbb{Q}_{1}} / Z^{\mathbb{Q}_{2}}$.

Proof. Denote by $Z^{\mathbb{Q}_{1}, \mathbb{Q}_{2}}$ the density process of $\mathbb{Q}_{1}$ with respect to $\mathbb{Q}_{2}$. We have

$$
Z_{T}^{\mathbb{Q}_{1}, \mathbb{Q}_{2}}:=\frac{\mathrm{d} \mathbb{Q}_{1}}{\mathrm{~d} \mathbb{Q}_{2}}=\frac{\mathrm{d} \mathbb{Q}_{1}}{\mathrm{dP}}\left(\frac{\mathrm{d} \mathbb{Q}_{2}}{\mathrm{dP}}\right)^{-1}=\frac{Z_{T}^{\mathbb{Q}_{1}}}{Z_{T}^{\mathbb{Q}_{2}}} .
$$

Hence, the $\mathbb{Q}_{2}$-martingale $Z^{\mathbb{Q}_{1}, \mathbb{Q}_{2}}$ is given by

$$
\begin{aligned}
Z_{t}^{\mathbb{Q}_{1}, \mathbb{Q}_{2}}=\mathbb{E}^{\mathbb{Q}_{2}}\left[Z_{T}^{\mathbb{Q}_{1}, \mathbb{Q}_{2}} \mid \mathcal{F}_{t}\right] & =\mathbb{E}^{\mathbb{Q}_{2}}\left[\frac{Z_{T}^{\mathbb{Q}_{1}}}{Z_{T}^{\mathbb{Q}_{2}}} \mid \mathcal{F}_{t}\right] \\
& =\frac{1}{Z_{t}^{\mathbb{Q}_{2}}} \mathbb{E}\left[Z_{T}^{\mathbb{Q}_{1}} \mid \mathcal{F}_{t}\right]=\frac{Z_{t}^{\mathbb{Q}_{1}}}{Z_{t}^{\mathbb{Q}_{2}}}, \quad 0 \leq t \leq T,
\end{aligned}
$$

the penultimate equality following from the Bayes rule applied between $\mathbb{Q}_{2}$ and $\mathbb{P}$, and the final equality from the fact that $Z^{\mathbb{Q}_{1}}$ is a $\mathbb{P}$-martingale.

A financial agent trades $S$ and has risk preferences represented by the exponential utility function

$$
U(x)=-\exp (-\alpha x), \quad \alpha>0, \quad x \in \mathbb{R},
$$

with risk aversion coefficient $\alpha$. A European contingent claim has $\mathcal{F}_{T}$-measurable payoff $F$. Following Becherer [2] and others, we assume that $F$ satisfies suitable exponential moment conditions:

$$
\mathbb{E}[\exp ((\alpha+\varepsilon) F)]<\infty, \quad \mathbb{E}[\exp (-\varepsilon F)]<\infty, \quad \text { for some } \varepsilon>0 .
$$

Condition (4.1) is sufficient to guarantee that $F$ is $\mathbb{Q}$-integrable for any $\mathbb{Q} \in \mathbf{M}_{f}$ (see for example Lemma A.1 in Becherer [2]). 
4.1. The dynamic primal and dual problems. The set $\Theta$ of admissible trading strategies is defined as the set of $S$-integrable processes $\theta$ such that the stochastic integral $\theta \cdot S$ is a $\mathbb{Q}$-martingale for every $\mathbb{Q} \in \mathbf{M}_{f}$, where $\theta$ is a $d$-dimensional vector representing the number of shares of each stock in the vector $S$. It is well-known 2, 11, 21, 37, 38 that there are a number of possible choices for a feasible set of permitted strategies, which all lead to the same value for the dual problem, defined further below, and it is on this latter problem that our analysis will be centred. For any $t \in[0, T]$, fix an $\mathcal{F}_{t}$-measurable random variable $x_{t}$, representing initial capital. Let $\Theta_{t}$ denote admissible strategies beginning at $t$.

The primal problem is to maximise expected utility of terminal wealth generated from trading $S$ and paying the claim payoff at $T$. The maximal expected utility process is

$$
u_{t}^{F}\left(x_{t}\right):=\underset{\theta \in \Theta_{t}}{\operatorname{ess} \sup _{0}} \mathbb{E}\left[-\mathrm{e}^{-\alpha\left(x_{t}+\int_{t}^{T} \theta_{u} \cdot \mathrm{d} S_{u}-F\right)} \mid \mathcal{F}_{t}\right], \quad 0 \leq t \leq T,
$$

with $\int_{t}^{T} \theta_{u} \cdot \mathrm{d} S_{u}=\sum_{i=1}^{d} \int_{t}^{T} \theta_{u}^{i} \mathrm{~d} S_{u}^{i}$.

We shall use the notational convention whereby setting $F=0$ in (4.2) signifies the corresponding quantity in the problem without the claim. Hence, the classical investment problem without the claim has maximal expected utility process $u^{0}$. Denote the optimiser in (4.2) by $\theta^{F}$, so $\theta^{0}$ is the optimiser in the problem without the claim.

The utility indifference price process for the claim, $p(\alpha)$, is defined by

$$
u_{t}^{F}\left(x_{t}+p_{t}(\alpha)\right)=u_{t}^{0}\left(x_{t}\right), \quad 0 \leq t \leq T .
$$

It is well-known (see for instance Becherer 2 or Mania and Schweizer 28]) that, with exponential utility, $p(\alpha)$ has no dependence on the starting capital (this follows from (4.2), where the initial capital factors out of the optimisation). The hedging strategy associated with this pricing mechanism is $\theta(\alpha)$, defined by

$$
\theta(\alpha):=\theta^{F}-\theta^{0} .
$$

The dual problem to (4.2) is defined by

$$
I_{t}^{F}:=\underset{\mathbb{Q} \in \mathbf{M}_{f}}{\operatorname{essinf}}\left[I_{t}(\mathbb{Q} \mid \mathbb{P})-\alpha \mathbb{E}^{\mathbb{Q}}\left[F \mid \mathcal{F}_{t}\right]\right], \quad 0 \leq t \leq T .
$$

Denote the optimiser in (4.3) by $\mathbb{Q}^{F}$, so the optimiser without the claim is $\mathbb{Q}^{0}$, the MEMM.

It is well-known (at least in a static context) that if we define the measure $\mathbb{P}_{F} \sim \mathbb{P}$ by

$$
\frac{\mathrm{d} \mathbb{P}_{F}}{\mathrm{~d} \mathbb{P}}:=\frac{\exp (\alpha F)}{\mathbb{E}[\exp (\alpha F)]},
$$

then we can use $\mathbb{P}_{F}$ instead of $\mathbb{P}$ as our reference measure, and this removes the claim from the primal and dual problems. In the dual picture, therefore, $\mathbb{Q}^{F}$ is the martingale measure which minimises the relative entropy between any $\mathbb{Q} \in \mathbf{M}_{f}$ and $\mathbb{P}_{F}$. These properties of $\mathbb{P}_{F}$ are wellknown in a static context from Delbaen et al [11]. The dynamic analogue of these arguments is given below.

Note that if we use $\mathbb{P}_{F}$ instead of $\mathbb{P}$ as reference measure, one could (in principle) define a set $\mathbf{M}_{f}\left(\mathbb{P}_{F}\right)$ of ELMMs with finite relative entropy with respect to $\mathbb{P}_{F}$, but it is well-known that $\mathbf{M}_{f}\left(\mathbb{P}_{F}\right)=\mathbf{M}_{f}(\mathbb{P})$ (see the statement and proof of Lemma A.1 in Becherer [2], for example) so we simply write $\mathbf{M}_{f}$.

Define the $\mathbb{P}$-martingale $M^{F}$ as the density process of $\mathbb{P}_{F}$ with respect to $\mathbb{P}$ :

$$
M_{t}^{F}:=\left.\frac{\mathrm{d} \mathbb{P}_{F}}{\mathrm{~d} \mathbb{P}}\right|_{\mathcal{F}_{t}}=\mathbb{E}\left[\frac{\mathrm{d} \mathbb{P}_{F}}{\mathrm{~d} \mathbb{P}} \mid \mathcal{F}_{t}\right]=\frac{\mathbb{E}\left[e^{\alpha F} \mid \mathcal{F}_{t}\right]}{\mathbb{E}\left[e^{\alpha F}\right]}, \quad 0 \leq t \leq T,
$$

which satisfies, for any integrable $\mathcal{F}_{T}$-measurable random variable $V$,

$$
\mathbb{E}^{\mathbb{P}_{F}}\left[V \mid \mathcal{F}_{t}\right]=\frac{1}{M_{t}^{F}} \mathbb{E}\left[M_{T}^{F} V \mid \mathcal{F}_{t}\right], \quad 0 \leq t \leq T .
$$


We "remove the claim" from the primal problem using the measure $\mathbb{P}_{F}$ as follows. Using (4.5) we convert (4.2) to

$$
u_{t}^{F}\left(x_{t}\right):=\mathbb{E}\left[e^{\alpha F} \mid \mathcal{F}_{t}\right] \underset{\theta \in \Theta_{t}}{\operatorname{ess} \sup _{t}} \mathbb{E}^{\mathbb{P}_{F}}\left[-\mathrm{e}^{-\alpha\left(x_{t}+\int_{t}^{T} \theta_{u} \cdot \mathrm{d} S_{u}\right)} \mid \mathcal{F}_{t}\right], \quad 0 \leq t \leq T,
$$

from which it is apparent that one may optimise over strategies in a problem without the claim and with $\mathbb{P}_{F}$ as reference measure. The same approach also works, of course, for the dual problem, as we show below. We shall need the following simple result relating the density process of any $\mathbb{Q} \in \mathbf{M}_{f}$ with respect to $\mathbb{P}$ to its counterpart with respect to $\mathbb{P}_{F}$.

Lemma 4.2. For any $\mathbb{Q} \in \mathbf{M}_{f}$, the density processes $Z^{\mathbb{Q}}$ and $Z^{\mathbb{Q}, \mathbb{P}_{F}}$ are related by

$$
Z_{t}^{\mathbb{Q}}=M_{t}^{F} Z_{t}^{\mathbb{Q}, \mathbb{P}_{F}}, \quad 0 \leq t \leq T .
$$

Proof. For $\mathbb{Q} \in \mathbf{M}_{f}$, we have

$$
\begin{aligned}
Z_{t}^{\mathbb{Q}, \mathbb{P}_{F}}=\mathbb{E}^{\mathbb{P}_{F}}\left[\frac{\mathrm{d} \mathbb{Q}}{\mathrm{d} \mathbb{P}_{F}} \mid \mathcal{F}_{t}\right] & =\mathbb{E}^{\mathbb{P}_{F}}\left[\frac{\mathrm{d} \mathbb{Q}}{\mathrm{dP}} / \frac{\mathrm{d} \mathbb{P}_{F}}{\mathrm{~d} \mathbb{P}} \mid \mathcal{F}_{t}\right] \\
& =\mathbb{E}^{\mathbb{P}_{F}}\left[\frac{1}{M_{T}^{F}} \frac{\mathrm{d} \mathbb{Q}}{\mathrm{d} \mathbb{P}} \mid \mathcal{F}_{t}\right] \\
& =\frac{1}{M_{t}^{F}} \mathbb{E}\left[\frac{\mathrm{d} \mathbb{Q}}{\mathrm{d} \mathbb{P}} \mid \mathcal{F}_{t}\right] \\
& =\frac{Z_{t}^{\mathbb{Q}}}{M_{t}^{F}}, \quad 0 \leq t \leq T,
\end{aligned}
$$

where we have used (4.5).

Applying Lemma 4.2 in turn at $t \leq T$ and at $T$, we obtain

$$
Z_{t, T}^{\mathbb{Q}, \mathbb{P}_{F}}=\frac{Z_{t, T}^{\mathbb{Q}}}{M_{t, T}^{F}}=\frac{\mathbb{E}\left[\mathrm{e}^{\alpha F} \mid \mathcal{F}_{t}\right]}{\mathrm{e}^{\alpha F}} Z_{t, T}^{\mathbb{Q}}, \quad 0 \leq t \leq T .
$$

We use this to "remove the claim" from the dual problem (4.3): compute, for any $\mathbb{Q} \in \mathbf{M}_{f}$,

$$
\begin{aligned}
I_{t}\left(\mathbb{Q} \mid \mathbb{P}_{F}\right) & =\mathbb{E}^{\mathbb{Q}}\left[\log Z_{t, T}^{\mathbb{Q}, \mathbb{P}_{F}} \mid \mathcal{F}_{t}\right] \\
& =I_{t}(\mathbb{Q} \mid \mathbb{P})-\alpha \mathbb{E}^{\mathbb{Q}}\left[F \mid \mathcal{F}_{t}\right]+\log \left(\mathbb{E}\left[\mathrm{e}^{\alpha F} \mid \mathcal{F}_{t}\right]\right), \quad 0 \leq t \leq T .
\end{aligned}
$$

Using this in (4.3), we obtain

$$
I_{t}^{F}=\underset{\mathbb{Q} \in \mathbf{M}_{f}}{\operatorname{essinf}}\left[I_{t}\left(\mathbb{Q} \mid \mathbb{P}_{F}\right)\right]-\log \left(\mathbb{E}\left[\mathrm{e}^{\alpha F} \mid \mathcal{F}_{t}\right]\right), \quad 0 \leq t \leq T .
$$

Since the last term on the right-hand-side does not depend on $\mathbb{Q}$, we see that we can reduce the dual problem to the problem

$$
I_{t}\left(\mathbb{Q} \mid \mathbb{P}_{F}\right) \longrightarrow \operatorname{min!}
$$

so that $\mathbb{Q}^{F}$ minimises $I\left(\mathbb{Q} \mid \mathbb{P}_{F}\right)$, and, when $F=0, \mathbb{Q}^{0}$ is the MEMM.

4.2. The fundamental duality. The duality results we need follow from the representation below for $Z^{\mathbb{Q}^{F}, \mathbb{P}_{F}}$, originally proven independently (to the best of our knowledge) by Grandits and Rheinländer [17] and Kabanov and Stricker [21] for $F=0$ (and hence for $Z^{\mathbb{Q}^{0}}$ ), but which applies equally well to $\mathbb{Q}^{F}$ if we use $\mathbb{P}_{F}$ as reference measure. Both [17] and [21] prove the result for a market involving a locally bounded semi-martingale $S$. This has been generalised to a general semi-martingale by Biagini and Frittelli [4]. 
Property 4.3 ([17, 21]). The density of the dual minimiser $\mathbb{Q}^{F}$ in 4.3) with respect to the measure $\mathbb{P}_{F}$ defined in (4.4) is given by

$$
\frac{\mathrm{d} \mathbb{Q}^{F}}{\mathrm{~d} \mathbb{P}_{F}} \equiv Z_{T}^{\mathbb{Q}^{F}, \mathbb{P}_{F}}=c_{F} \exp \left(-\alpha\left(\theta^{F} \cdot S\right)_{T}\right), \quad c_{F} \in \mathbb{R}_{+},
$$

where $\theta^{F} \in \Theta$ is the optimal strategy in the primal problem 4.2) and the stochastic integral $\left(\theta^{F} \cdot S\right)$ is a $\mathbb{Q}$-martingale for any $\mathbb{Q} \in \mathbf{M}_{f}$.

We convert this to the dynamic result below, in which we also restore $\mathbb{P}$ as reference measure.

Corollary 4.4. The density process $Z^{\mathbb{Q}^{F}}$ of the dual minimiser $\mathbb{Q}^{F}$ in 4.3) satisfies, for $t \in[0, T]$,

$$
Z_{t, T}^{\mathbb{Q}^{F}}=\exp \left[I_{t}\left(\mathbb{Q}^{F} \mid \mathbb{P}\right)-\alpha\left(\mathbb{E}^{\mathbb{Q}^{F}}\left[F \mid \mathcal{F}_{t}\right]+\int_{t}^{T} \theta_{u}^{F} \cdot \mathrm{d} S_{u}-F\right)\right]
$$

where $\theta^{F} \in \Theta$ is the optimal strategy in the primal problem (4.2).

Proof. First, we obtain a dynamic version of (4.8). Using (4.8) and the $\mathbb{Q}^{F}$-martingale property of $\left(\theta^{F} \cdot S\right)$, we have

$$
\begin{aligned}
I_{t}\left(\mathbb{Q}^{F} \mid \mathbb{P}_{F}\right) & =\mathbb{E}^{\mathbb{Q}^{F}}\left[\log Z_{t, T}^{\mathbb{Q}^{F}, \mathbb{P}_{F}} \mid \mathcal{F}_{t}\right] \\
& =\mathbb{E}^{\mathbb{Q}^{F}}\left[\log c_{F}-\alpha\left(\theta^{F} \cdot S\right)_{T} \mid \mathcal{F}_{t}\right]-\log Z_{t}^{\mathbb{Q}^{F}, \mathbb{P}_{F}} \\
& =\log c_{F}-\alpha\left(\theta^{F} \cdot S\right)_{t}-\log Z_{t}^{\mathbb{Q}^{F}, \mathbb{P}_{F}}, \quad 0 \leq t \leq T .
\end{aligned}
$$

Using this in turn at $t \leq T$ and $T$ we obtain

$$
Z_{t, T}^{\mathbb{Q}^{F}, \mathbb{P}_{F}}=c_{t}^{F} \exp \left(-\alpha \int_{t}^{T} \theta_{u}^{F} \cdot \mathrm{d} S_{u}\right), \quad c_{t}^{F}:=\exp \left(I_{t}\left(\mathbb{Q}^{F} \mid \mathbb{P}_{F}\right), \quad 0 \leq t \leq T,\right.
$$

which is a dynamic version of (4.8). Using this along with (4.6) and (4.7) we obtain

$$
Z_{t, T}^{\mathbb{Q}^{F}}=\exp \left(I_{t}^{F}-\alpha \int_{t}^{T} \theta_{u}^{F} \cdot \mathrm{d} S_{u}+\alpha F\right), \quad 0 \leq t \leq T .
$$

Finally, using the definition (4.3) of $I^{F}$ gives the result.

Corollary 4.4 is nothing more than a dynamic version of the classical result of Grandits and Rheinländer [17] and Kabanov and Stricker 21] for the MEMM, with the added generalisation of allowing for $\mathbb{P}_{F}$ as reference measure. It leads immediately to the duality result below, a dynamic version of the duality in Delbaen et al [11. This result is stated in Mania and Schweizer [28] for a bounded claim. We give a proof to highlight that the boundedness condition on the claim is not needed.

Theorem 4.5 ([11, 2, 21, 28]). Suppose the claim payoff $F$ satisfies the exponential moment conditions (4.1). Then the maximal expected utility process in (4.2) and the optimal dual process in (4.3) are related by

$$
u_{t}^{F}\left(x_{t}\right)=-\exp \left(-\alpha x_{t}-I_{t}^{F}\right), \quad 0 \leq t \leq T .
$$

Proof. We compute the primal optimal expected utility process and use Corollary 4.4 to substitute for the stochastic integral $\left(\theta^{F} \cdot S\right)$ :

$$
\begin{aligned}
u_{t}^{F}\left(x_{t}\right) & =\mathbb{E}\left[-\mathrm{e}^{-\alpha\left(x_{t}+\int_{t}^{T} \theta_{u}^{F} \cdot \mathrm{d} S_{u}-F\right)} \mid \mathcal{F}_{t}\right] \\
& \left.=-\mathrm{e}^{-\alpha x_{t}} \mathbb{E}\left[Z_{t, T}^{\mathbb{Q}^{F}} \exp \left(-I_{t}^{F}\right) \mid \mathcal{F}_{t}\right] \quad \text { (using Corollary 4.4 }\right) \\
& =-\exp \left(-\alpha x_{t}-I_{t}^{F}\right), \quad 0 \leq t \leq T .
\end{aligned}
$$


Using this theorem and the definition of the indifference price we obtain the following dual representation of the indifference price process, a dynamic version of the classical representation.

Corollary 4.6. The indifference price process has the dual representation

$$
p_{t}(\alpha)=-\frac{1}{\alpha}\left(I_{t}^{F}-I_{t}^{0}\right), \quad 0 \leq t \leq T .
$$

Written out explicitly, (4.11) can be re-cast into the more familiar form

$$
p_{t}(\alpha)=\operatorname{esssup}_{\mathbb{Q} \in \mathbf{M}_{f}}\left[\mathbb{E}^{\mathbb{Q}}\left[F \mid \mathcal{F}_{t}\right]-\frac{1}{\alpha}\left(I_{t}(\mathbb{Q} \mid \mathbb{P})-I_{t}\left(\mathbb{Q}^{0} \mid \mathbb{P}\right)\right)\right], \quad 0 \leq t \leq T .
$$

The two conditional entropy terms in (4.12) can in fact be condensed into one, using the following proposition.

Proposition 4.7. The conditional entropy process I satisfies the property that, for any equivalent local martingale measure $\mathbb{Q} \in \mathbf{M}_{f}$,

$$
I_{t}(\mathbb{Q} \mid \mathbb{P})-I_{t}\left(\mathbb{Q}^{0} \mid \mathbb{P}\right)=I_{t}\left(\mathbb{Q} \mid \mathbb{Q}^{0}\right), \quad 0 \leq t \leq T .
$$

Proof. For any $\mathbb{Q} \in \mathbf{M}_{f}$, the conditional entropy process $I\left(\mathbb{Q} \mid \mathbb{Q}^{0}\right)$ is given by

$$
\begin{aligned}
I_{t}\left(\mathbb{Q} \mid \mathbb{Q}^{0}\right) & :=\mathbb{E}^{\mathbb{Q}}\left[\log Z_{t, T}^{\mathbb{Q}, \mathbb{Q}^{0}} \mid \mathcal{F}_{t}\right] \\
& =\mathbb{E}^{\mathbb{Q}}\left[\log Z_{t, T}^{\mathbb{Q}}-\log Z_{t, T}^{\mathbb{Q}^{0}} \mid \mathcal{F}_{t}\right] \\
& =I_{t}(\mathbb{Q} \mid \mathbb{P})-\mathbb{E}^{\mathbb{Q}}\left[\log Z_{t, T}^{\mathbb{Q}^{0}} \mid \mathcal{F}_{t}\right], \quad 0 \leq t \leq T .
\end{aligned}
$$

We have the dynamic version of the Grandits-Rheinländer [17] representation of the MEMM, given by (4.9) for $F=0$ :

$$
Z_{t, T}^{\mathbb{Q}^{0}}=\exp \left(I_{t}\left(\mathbb{Q}^{0} \mid \mathbb{P}\right)-\alpha \int_{t}^{T} \theta_{u}^{0} \cdot \mathrm{d} S_{u}\right), \quad 0 \leq t \leq T,
$$

where the optimal investment strategy $\theta^{0} \in \Theta$, so $\left(\theta^{0} \cdot S\right)$ is a $\mathbb{Q}$-martingale, for any $\mathbb{Q} \in \mathbf{M}_{f}$. Using this in (4.14) we obtain (4.13).

Using Proposition 4.7 in the classical dual stochastic control representation (4.11) of the indifference price process, we immediately obtain the following form for $p(\alpha)$, which will form the basis for our asymptotic expansion of the indifference price process.

Lemma 4.8. The indifference price process is given by the dual stochastic control representation

$$
p_{t}(\alpha)=\operatorname{esssup}_{\mathbb{Q} \in \mathbf{M}_{f}}\left[\mathbb{E}^{\mathbb{Q}}\left[F \mid \mathcal{F}_{t}\right]-\frac{1}{\alpha} I_{t}\left(\mathbb{Q} \mid \mathbb{Q}^{0}\right)\right], \quad 0 \leq t \leq T .
$$

Proof. Use (4.13) in (4.11).

Remark 4.9. A version of Lemma 4.8 for American claims was given in Leung et al [26] in a stochastic volatility scenario (see their Proposition 7).

Remark 4.10. The optimiser in Lemma 4.8 is also the optimiser in (4.12), that is, $\mathbb{Q}^{F}$.

\section{INDIFFERENCE VALUATION IN AN INCOMPLETE ITÔ PROCESS MARKET}

In this section we apply the indifference pricing formula from Lemma 4.8 in an Itô process setting, and we show how it leads to a control problem of a similar structure to the one analysed in Section 3 .

We have a probability space $(\Omega, \mathcal{F}, \mathbb{P})$ equipped with the standard augmented filtration $\mathbb{F}:=$ $\left(\mathcal{F}_{t}\right)_{0 \leq t \leq T}$ associated with an $m$-dimensional Brownian motion $W$. On this space we have a 
financial market with (for simplicity) zero interest rate. The price processes of $d<m$ stocks are given by the vector $S=\left(S^{1}, \ldots, S^{d}\right)^{\top}$, where $S=\left(S_{t}\right)_{0 \leq t \leq T}$ follows the Itô process

$$
\mathrm{d} S_{t}=\operatorname{diag}_{d}\left(S_{t}\right)\left[\mu_{t}^{S} \mathrm{~d} t+\sigma_{t} \mathrm{~d} W_{t}\right]
$$

with $\operatorname{diag}_{d}(\cdot)$ denoting the $(d \times d)$ matrix with zero entries off the main diagonal. The $d$-dimensional appreciation rate vector $\mu^{S}$ and the $(d \times m)$ volatility matrix $\sigma$ are $\mathbb{F}$-progressively measurable processes satisfying $\int_{0}^{T}\left\|\mu_{t}^{S}\right\| \mathrm{d} t<\infty$ and $\int_{0}^{T} \sigma_{t} \sigma_{t}^{\top} \mathrm{d} t<\infty$, almost surely. The volatility matrix $\sigma_{t}$ has full rank for every $t \in[0, T]$, so that the matrix $\left(\sigma_{t} \sigma_{t}^{\top}\right)^{-1}$ is well-defined, as is the $m$ dimensional relative risk process given by

$$
\lambda_{t}:=\sigma_{t}^{\top}\left(\sigma_{t} \sigma_{t}^{\top}\right)^{-1} \mu_{t}^{S}, \quad 0 \leq t \leq T .
$$

For $d<m$, this market is incomplete. We also have a vector $Y=\left(Y^{1}, \ldots, Y^{m-d}\right)^{\top}$ of $(m-d)$ non-traded factors. These could be the prices of non-traded assets, or of factors such as stochastic volatilities and correlations. This framework is general enough to encompass multi-dimensional versions of basis risk models as well as multi-factor stochastic volatility models, with no Markovian structure needed. We assume that $Y$ follows the Itô process

$$
\mathrm{d} Y_{t}=\operatorname{diag}_{m-d}\left(Y_{t}\right)\left[\mu_{t}^{Y} \mathrm{~d} t+\beta_{t} \mathrm{~d} W_{t}\right],
$$

for an $(m-d)$-dimensional progressively measurable vector $\mu^{Y}$ satisfying $\int_{0}^{T}\left\|\mu_{t}^{Y}\right\| \mathrm{d} t<\infty$, almost surely, and an $(m-d) \times m$-dimensional progressively measurable matrix $\beta$ satisfying $\int_{0}^{T} \beta_{t} \beta_{t}^{\top} \mathrm{d} t<$ $\infty$, almost surely.

A European contingent claim has $\mathcal{F}_{T}$-measurable payoff $F$ depending on the evolution of $(S, Y)$. We assume $F$ satisfies Assumption 2.1, so in particular, $F \in L^{2}(\mathbb{Q})$, for any ELMM $\mathbb{Q} \in \mathbf{M}_{f}$.

Measures $\mathbb{Q} \sim \mathbb{P}$ have density processes with respect to $\mathbb{P}$ of the form

$$
Z_{t}^{\mathbb{Q}}=\mathcal{E}(-q \cdot W)_{t}, \quad 0 \leq t \leq T,
$$

for some $m$-dimensional process $q$ satisfying $\int_{0}^{T}\left\|q_{t}\right\|^{2} \mathrm{~d} t<\infty$ almost surely. For $Z^{\mathbb{Q}}$ to be the density of an equivalent local martingale measure, it must be is a $\mathbb{P}$-martingale (a Novikov condition on $q$ would guarantee this) and in addition $q$ must satisfy

$$
\mu_{t}^{S}-\sigma_{t} q_{t}=\mathbf{0}_{d}, \quad 0 \leq t \leq T
$$

where $\mathbf{0}_{d}$ denotes the $d$-dimensional zero vector, so that $S$ is a local $\mathbb{Q}$-martingale.

As the market is incomplete, there will be an infinite number of solutions $q$ to the equations (5.4), and the ELMMs $\mathbb{Q}$ are in one-to-one correspondence with processes $q$ satisfying (5.4) and such that $\mathcal{E}(-q \cdot W)$ is a $\mathbb{P}$-martingale.

By the Girsanov theorem, the process $W^{\mathbb{Q}}$ defined by

$$
W_{t}^{\mathbb{Q}}:=W_{t}+\int_{0}^{t} q_{u} \mathrm{~d} u, \quad 0 \leq t \leq T,
$$

is an $m$-dimensional $\mathbb{Q}$-Brownian motion. The dynamics of the stocks and non-traded factors under $\mathbb{Q}$ are then

$$
\begin{aligned}
\mathrm{d} S_{t} & =\operatorname{diag}_{d}\left(S_{t}\right) \sigma_{t} \mathrm{~d} W_{t}^{\mathbb{Q}}, \\
\mathrm{d} Y_{t} & =\operatorname{diag}_{m-d}\left(Y_{t}\right)\left[\left(\mu_{t}^{Y}-\beta_{t} q_{t}\right) \mathrm{d} t+\beta_{t} \mathrm{~d} W_{t}^{\mathbb{Q}}\right] .
\end{aligned}
$$

If we choose $q=\lambda$, given by (5.2), we obtain the minimal martingale measure $\mathbb{Q}_{M}$, while the density process of the MEMM $\mathbb{Q}^{0}$ is $Z^{\mathbb{Q}^{0}}=\mathcal{E}\left(-q^{0} \cdot W\right)$, for some integrand $q^{0}$.

Denote by $H^{2}(\mathbb{Q})$ the space of $L^{2}$-bounded continuous $\mathbb{Q}$-martingales $M\left(\operatorname{so}, \sup _{t \in[0, T]} \mathbb{E}^{\mathbb{Q}}\left[M_{t}^{2}\right]<\right.$ $\infty)$. By Proposition IV.1.23 and Corollary IV.1.25 in Revuz and Yor $\left[35, H^{2}(\mathbb{Q})\right.$ is also the space of martingales $M$ such that $\mathbb{E}^{\mathbb{Q}}\left[[M]_{T}\right]<\infty$. Denoting $\Lambda^{\mathbb{Q}}:=\left(q \cdot W^{\mathbb{Q}}\right)$, then using (5.3) and (5.5), $\log Z^{\mathbb{Q}}=-\Lambda^{\mathbb{Q}}+\left[\Lambda^{\mathbb{Q}}\right] / 2$, so the relative entropy between $\mathbb{Q} \in \mathbf{M}_{f}$ and $\mathbb{P}$ is given by

$$
0 \leq I_{0}(\mathbb{Q} \mid \mathbb{P})=\mathbb{E}^{\mathbb{Q}}\left[-\Lambda_{T}^{\mathbb{Q}}+\frac{1}{2}\left[\Lambda^{\mathbb{Q}}\right]_{T}\right]<\infty,
$$


the last inequality true by assumption. The finiteness and non-negativity of this relative entropy yields that both expectations above are finite. Precisely, we have $\mathbb{E}^{\mathbb{Q}}\left[\Lambda_{T}^{\mathbb{Q}}\right]>-\infty$ and, in particular, $\mathbb{E}^{\mathbb{Q}}\left[\left[\Lambda^{\mathbb{Q}}\right]_{T}\right]<\infty$, the latter condition implying that $\Lambda^{\mathbb{Q}} \in H^{2}(\mathbb{Q})$. Therefore,

$$
\Lambda^{\mathbb{Q}}:=\left(q \cdot W^{\mathbb{Q}}\right) \text { is a } \mathbb{Q} \text {-martingale, for all } \mathbb{Q} \in \mathbf{M}_{f} .
$$

This will be useful in computing the conditional relative entropy $I\left(\mathbb{Q}^{0} \mathbb{Q}^{0}\right)$.

Using (5.5) in turn for $\mathbb{Q}$ and $\mathbb{Q}^{0}$, we have

$$
W_{t}^{\mathbb{Q}}=W_{t}^{\mathbb{Q}^{0}}+\int_{0}^{t}\left(q_{t}-q_{t}^{0}\right) \mathrm{d} t, \quad 0 \leq t \leq T,
$$

where $W^{\mathbb{Q}^{0}}$ is a $\mathbb{Q}^{0}$-Brownian motion.

Note that since both $q$ and $q^{0}$ satisfy (5.4), we have

$$
\sigma_{t}\left(q_{t}-q_{t}^{0}\right)=\mathbf{0}_{d}, \quad 0 \leq t \leq T,
$$

which we shall use later.

Using (5.9), we can write the $\mathbb{Q}$-dynamics of $Y$ in (5.7) as

$$
\mathrm{d} Y_{t}=\operatorname{diag}_{m-d}\left(Y_{t}\right)\left[\left(\mu_{t}^{Y}-\beta_{t} q_{t}^{0}\right) \mathrm{d} t+\beta_{t}\left(\mathrm{~d} W_{t}^{\mathbb{Q}}-\left(q_{t}-q_{t}^{0}\right) \mathrm{d} t\right)\right] .
$$

The point of this representation is that the $\mathbb{Q}$-dynamics of $Y$ may be interpreted as a perturbation of the $\mathbb{Q}^{0}$-dynamics, since setting $q=q^{0}$ gives the dynamics under the MEMM $\mathbb{Q}^{0}$, with the Brownian motion $W^{\mathbb{Q}}$ also being modulated by the choice of $q$.

Using (5.5) and (5.9), the density process of $\mathbb{Q}$ with respect to $\mathbb{Q}^{0}$ is

$$
Z_{t}^{\mathbb{Q}, \mathbb{Q}^{0}}=\frac{Z_{t}^{\mathbb{Q}}}{Z_{t}^{\mathbb{Q}^{0}}}=\frac{\mathcal{E}(-q \cdot W)_{t}}{\mathcal{E}\left(-q^{0} \cdot W\right)_{t}}=\mathcal{E}\left(-\left(q-q^{0}\right) \cdot W^{\mathbb{Q}^{0}}\right)_{t}, \quad 0 \leq t \leq T .
$$

Using this, along with (5.9) and the martingale condition (5.8), we compute

$$
I_{t}\left(\mathbb{Q} \mid \mathbb{Q}^{0}\right)=\mathbb{E}^{\mathbb{Q}}\left[\frac{1}{2} \int_{t}^{T}\left\|q_{u}-q_{u}^{0}\right\|^{2} \mathrm{~d} u \mid \mathcal{F}_{t}\right], \quad 0 \leq t \leq T .
$$

Now we explicitly consider $\mathbb{Q}$ as a perturbation around $\mathbb{Q}^{0}$. Introduce, for some small parameter $\varepsilon$, a parametrised family of measures $\{\mathbb{Q}(\varepsilon)\}_{\varepsilon \in \mathbb{R}}$, such that

$$
\mathbb{Q} \equiv \mathbb{Q}(\varepsilon), \quad \mathbb{Q}^{0} \equiv \mathbb{Q}(0),
$$

and also write

$$
q-q^{0}=:-\varepsilon \varphi
$$

for some process $\varphi$. Then (5.10) becomes

$$
\sigma \varphi=\mathbf{0}_{d}
$$

Denote by $\mathcal{A}\left(\mathbf{M}_{f}\right)$ the set of such $\varphi$ which correspond to $\mathbb{Q} \in \mathbf{M}_{f}$, and also define the process $\Phi:=\int_{0}^{\cdot} \varphi_{s} \mathrm{~d} s$.

The $\mathbb{Q}(\varepsilon)$-dynamics of the state variables $S, Y$ in this notation are then

$$
\begin{aligned}
\mathrm{d} S_{t} & =\operatorname{diag}_{d}\left(S_{t}\right) \sigma_{t} \mathrm{~d} W_{t}^{\mathbb{Q}(\varepsilon)}, \\
\mathrm{d} Y_{t} & =\operatorname{diag}_{m-d}\left(Y_{t}\right)\left[\left(\mu_{t}^{Y}-\beta_{t} q_{t}^{0}\right) \mathrm{d} t+\beta_{t}\left(\mathrm{~d} W_{t}^{\mathbb{Q}(\varepsilon)}+\varepsilon \varphi_{t} \mathrm{~d} t\right)\right] .
\end{aligned}
$$

Observe that if we define the state variable $X:=(S, Y)^{\top}$, then we have recovered dynamics of the general form (3.3).

The $\mathbb{Q}(\varepsilon)$-dynamics (5.15) of $S$, along with the constraint (5.14), lead to the following orthogonality result between trading strategies and dual controls. Consider integrands $\theta^{(\varepsilon)}, \varphi$ such that $\left(\theta^{(\varepsilon)} \cdot S\right)$ is a $\mathbb{Q}(\varepsilon)$-martingale and $\varphi$ satisfies (5.14). Then a straightforward computation using (5.15) and (5.14) shows that the stochastic integrals $\left(\theta^{(\varepsilon)} \cdot S\right)$ and $\left(\varphi \cdot W^{\mathbb{Q}(\varepsilon)}\right)$ are orthogonal $\mathbb{Q}(\varepsilon)$-martingales. That is, $\mathbb{E}^{\mathbb{Q}(\varepsilon)}\left[\left(\theta^{(\varepsilon)} \cdot S\right)_{T}\left(\varphi \cdot W^{\mathbb{Q}(\varepsilon)}\right)_{T}\right]=0$. In particular, this will hold for $\varepsilon=0$. 
A similar orthogonality result is reflected in the following decomposition of the claim payoff $F$. When the dynamics of the state variables are given as in (5.15) and (5.16), we write $F \equiv$ $F\left(W^{\mathbb{Q}(\varepsilon)}+\varepsilon \Phi\right)$. Write the Galtchouk-Kunita-Watanabe decomposition of $F$ under $\mathbb{Q}(0) \equiv \mathbb{Q}^{0}$ as

$$
F\left(W^{\mathbb{Q}(0)}\right)=\mathbb{E}^{\mathbb{Q}(0)}\left[F\left(W^{\mathbb{Q}(0)}\right)\right]+\left(\theta^{(0)} \cdot S\right)_{T}+\left(\xi^{(0)} \cdot W^{\mathbb{Q}(0)}\right)_{T},
$$

for some integrands $\theta^{(0)}, \xi^{(0)}$, such that the stochastic integrals in (5.17) are orthogonal $\mathbb{Q}(0)$ martingales, so we have

$$
\mathbb{E}^{\mathbb{Q}(0)}\left[\left(\theta^{(0)} \cdot S\right)_{T}\left(\xi^{(0)} \cdot W^{\mathbb{Q}(0)}\right)_{T}\right]=0 .
$$

Using (5.11) and (5.13), the indifference price process, as given by Lemma 4.8, has the stochastic control representation

$$
p_{t}(\alpha)=\sup _{\varphi \in \mathcal{A}\left(\mathbf{M}_{f}\right)} \mathbb{E}^{\mathbb{Q}(\varepsilon)}\left[F\left(W^{\mathbb{Q}(\varepsilon)}+\varepsilon \Phi\right)-\frac{\varepsilon^{2}}{2 \alpha} \int_{t}^{T}\left\|\varphi_{u}\right\|^{2} \mathrm{~d} u \mid \mathcal{F}_{t}\right], \quad 0 \leq t \leq T .
$$

If we choose

$$
\varepsilon^{2}=\alpha,
$$

then we get a control problem of the form

$$
p_{t}(\alpha)=\sup _{\varphi \in \mathcal{A}\left(\mathbf{M}_{f}\right)} \mathbb{E}^{\mathbb{Q}(\varepsilon)}\left[F\left(W^{\mathbb{Q}(\varepsilon)}+\varepsilon \int_{t}^{\cdot} \varphi_{u} \mathrm{~d} u\right)-\frac{1}{2} \int_{t}^{T}\left\|\varphi_{u}\right\|^{2} \mathrm{~d} u \mid \mathcal{F}_{t}\right], \quad 0 \leq t \leq T .
$$

subject to $\mathbb{Q}(\varepsilon)$-dynamics of $S, Y$ given by (5.15), (5.16), and with $\mathbb{Q}(0)$ corresponding to the MEMM $\mathbb{Q}^{0}$. We have now formulated the indifference pricing control problem in the form of a control problem akin to that described in Section 3 . We then have the following result.

Theorem 5.1. Let the payoff of the claim, $F$, be a functional of the paths of $S, Y$, satisfying Assumption 2.1. Let the $\mathbb{Q}(\varepsilon)$-dynamics of $S, Y$ be given by (5.15,5.16), with $\mathbb{Q}(\varepsilon)$ given by (5.12), and with the parameter $\varepsilon$ given by (5.18). Then for small risk aversion $\alpha$, the indifference price process of the claim has the asymptotic expansion

$$
p_{t}(\alpha)=\mathbb{E}^{\mathbb{Q}^{0}}\left[F \mid \mathcal{F}_{t}\right]+\frac{1}{2} \alpha \mathbb{E}^{\mathbb{Q}^{0}}\left[\int_{t}^{T}\left\|\xi_{u}^{(0)}\right\|^{2} \mathrm{~d} u \mid \mathcal{F}_{t}\right]+O\left(\alpha^{2}\right), \quad 0 \leq t \leq T,
$$

where $\mathbb{Q}^{0}$ is the minimal entropy martingale measure, and $\xi^{(0)}$ is the process in the KunitaWatanabe decomposition (5.17) of the claim, under $\mathbb{Q}(0) \equiv \mathbb{Q}^{0}$.

Proof. In the state dynamics (5.1515.16) each choice of the perturbation $\varepsilon \varphi$ gives rise to a different measure $\mathbb{Q}(\varepsilon)$. To apply Theorem 3.1, we fix a measure $\mathbb{M}$ and instead consider the perturbed state process $X^{(\varepsilon)}=\left(S^{(\varepsilon)}, Y^{(\varepsilon)}\right)^{\top}$, with dynamics under $\mathbb{M}$ given by

$$
\begin{aligned}
\mathrm{d} S_{t}^{(\varepsilon)} & =\operatorname{diag}_{d}\left(S_{t}^{(\varepsilon)}\right) \sigma_{t} \mathrm{~d} W_{t}^{\mathbb{M}}, \\
\mathrm{d} Y_{t}^{(\varepsilon)} & =\operatorname{diag}_{m-d}\left(Y_{t}^{(\varepsilon)}\right)\left[\left(\mu_{t}^{Y}-\beta_{t} q_{t}^{0}\right) \mathrm{d} t+\beta_{t}\left(\mathrm{~d} W_{t}^{\mathbb{M}}+\varepsilon \varphi_{t} \mathrm{~d} t\right)\right],
\end{aligned}
$$

for some $m$-dimensional $\mathbb{M}$-Brownian motion $W^{\mathbb{M}}$. The dynamics of the state variable $X^{(\varepsilon)}$ under $\mathbb{M}$ match those of $(S, Y)^{\top}$ under $\mathbb{Q}(\varepsilon)$, and are of the required form (3.3), with $\varepsilon=0$ corresponding to the MEMM $\mathbb{Q}^{0}$. We can now apply Theorem 3.1 directly, with the KunitaWatanabe decomposition (5.17) of the claim under $\mathbb{Q}(0) \equiv \mathbb{Q}^{0}$ taking the place of the martingale representation result (2.8), and the result duly follows.

The underlying message of Theorem 5.1 is that for small risk aversion, the lowest order contribution to the indifference price process is the marginal utility-based price process $\widehat{p}_{t}:=\mathbb{E}^{\mathbb{Q}^{0}}\left[F \mid \mathcal{F}_{t}\right]$, corresponding to the valuation methodology developed by Davis 9 . The first order correction is a mean-variance correction, since the Kunita-Watanabe decomposition (5.17) for $\varepsilon=0$ is the Föllmer-Schweizer-Sondermann decomposition of the claim under $\mathbb{Q}^{0}$, and the integrand $\theta^{(0)}$ in 
(5.17) is a risk-minimising strategy in the sense of Föllmer and Sondermann [13] under $\mathbb{Q}^{0}$. Similar results have been obtained for bounded claims by Mania and Schweizer [28] and Kallsen and Rheinländer [23]. The contribution here is to show a new methodology for obtaining this result, for a square-integrable claim. The strategy $\theta^{(0)}$ is, in general, the zero risk aversion limit of the optimal hedging strategy $\theta(\alpha)$ (see, for example, [28, 23] for a bounded claim), and hence can also be interpreted as the marginal utility-based hedging strategy.

Note that using (5.17) for $\varepsilon=0$, we can write (5.19) as

$$
p_{t}(\alpha)=\mathbb{E}^{\mathbb{Q}^{0}}\left[F \mid \mathcal{F}_{t}\right]+\frac{1}{2} \alpha\left(\operatorname{var}^{\mathbb{Q}^{0}}\left[F \mid \mathcal{F}_{t}\right]-\mathbb{E}^{\mathbb{Q}^{0}}\left[\int_{t}^{T}\left\|\theta_{u}^{(0)}\right\|^{2} \mathrm{~d}[S]_{u} \mid \mathcal{F}_{t}\right]\right)+O\left(\alpha^{2}\right),
$$

for $t \in[0, T]$, which highlights the mean-variance structure of the asymptotic representation.

\section{Applications}

Here we show some examples where Theorem 5.1 would apply. In these examples we assume that the functional $F$ satisfies Assumption 2.1. This is a relatively mild assumption and would apply in a wide range of models, but of course would need to be checked on a case-by-case basis in specific models, and would depend on the model and also on the specific form of the functional $F$. We give a concrete case in Example 6.2 of a lookback put option on a non-traded asset, where we check that Assumption 2.1 is satisfied.

Example 6.1 (Multi-dimensional random parameter basis risk model). This is the model of Section 5. with $d$ traded stocks $S$ and $(m-d)$ non-traded assets $Y$, and with the volatility process $\sigma$ in (5.1) given by

$$
\sigma_{t}=\left(\begin{array}{cc}
\sigma_{t}^{S} & \mathbf{0}_{d \times(m-d)}
\end{array}\right), \quad 0 \leq t \leq T,
$$

where $\sigma^{S}$ is a $d \times d$ invertible matrix process, and where $\mathbf{0}_{d \times(m-d)}$ denotes the zero $d \times(m-d)$ matrix. Write the $m$-dimensional Brownian motion $W$ as $W=\left(W^{S}, W^{S, \perp}\right)^{\top}$, where $W^{S}$ denotes the first $d$ components of $W$. Then the $d$ traded stocks are driven by $d$ Brownian motions, and the non-traded assets are imperfectly correlated with $S$. The claim payoff $F$ is typically dependent on the evolution of $Y$ only, though our results are valid without this restriction.

In this case, the process $\lambda$ in (5.2) and the integrand $q$ in (5.3) are given by

$$
\lambda_{t}=\left(\begin{array}{c}
\lambda_{t}^{S} \\
\mathbf{0}_{m-d}
\end{array}\right), \quad q_{t}=\left(\begin{array}{c}
\lambda_{t}^{S} \\
\gamma_{t}
\end{array}\right), \quad 0 \leq t \leq T
$$

where $\lambda^{S}$ is the stocks' $d$-dimensional market price of risk process, given by $\lambda^{S}:=\left(\sigma^{S}\right)^{-1} \mu^{S}$, and $\gamma$ is an $(m-d)$-dimensional adapted process. Each choice of $\gamma$ leads to a different ELMM $\mathbb{Q}$, with $\gamma=\mathbf{0}_{m-d}$ corresponding to the minimal martingale measure $\mathbb{Q}_{M}$, and $\gamma=\gamma^{0}$ corresponding to the minimal entropy martingale measure $\mathbb{Q}^{0} \equiv \mathbb{Q}_{E}$, for some $(m-d)$-dimensional process $\gamma^{0}$. The density process of any ELMM $\mathbb{Q} \in \mathbf{M}_{f}$ is then given by

$$
Z_{t}^{\mathbb{Q}}=\mathcal{E}\left(-\lambda^{S} \cdot W^{S}-\gamma \cdot W^{S, \perp}\right)_{t}, \quad 0 \leq t \leq T .
$$

The indifference price expansion of the claim with payoff $F$ is then of the form (5.19) or, equivalently, (5.20).

A special feature of these models arises when the process $\lambda^{S}$ is either deterministic or does not depend on the non-traded asset prices $Y$. In this case it is not hard to see that the MEMM $\mathbb{Q}^{0}=\mathbb{Q}_{M}$. This is because the relative entropy process between $\mathbb{Q} \in \mathbf{M}_{f}$ and $\mathbb{P}$ is given by

$$
I_{t}(\mathbb{Q} \mid \mathbb{P})=\mathbb{E}^{\mathbb{Q}}\left[\frac{1}{2} \int_{t}^{T}\left(\left\|\lambda_{u}^{S}\right\|^{2}+\left\|\gamma_{u}\right\|^{2}\right) \mathrm{d} u \mid \mathcal{F}_{t}\right], \quad 0 \leq t \leq T .
$$

The problem of finding the minimal entropy martingale measure is then to minimise this functional subject to $\mathbb{Q}$-dynamics of $S, Y$ given by (5.65.7), with the process $\gamma$ playing the role of a control. 
In the current notation, the $\mathbb{Q}$-dynamics of $Y$ are

$$
\mathrm{d} Y_{t}=\operatorname{diag}_{m-d}\left(Y_{t}\right)\left[\left(\mu_{t}^{Y}-\beta_{t}\left(\begin{array}{c}
\lambda_{t}^{S} \\
\gamma_{t}
\end{array}\right)\right) \mathrm{d} t+\beta_{t} \mathrm{~d} W_{t}^{\mathbb{Q}}\right] .
$$

From this it is clear that if $\lambda^{S}$ does not depend on $Y$, then it is unaffected by the control, and then the relative entropy process in (6.2) is minimised by choosing $\gamma=\mathbf{0}_{m-d}$, so $\mathbb{Q}_{E} \equiv \mathbb{Q}^{0}=\mathbb{Q}_{M}$. In this case, the Kunita-Watanabe decomposition of the claim under $\mathbb{Q}_{M}$ will be of the form

$$
F=\mathbb{E}^{\mathbb{Q}_{M}}[F]+\left(\theta^{M} \cdot S\right)_{T}+\left(\xi^{M} \cdot W^{\mathbb{Q}_{M}}\right)_{T},
$$

for integrands $\theta^{M}, \xi^{M}$ such that the $\mathbb{Q}_{M}$-martingales $\left(\theta^{M} \cdot S\right)$ and $\left(\xi^{M} \cdot W^{\mathbb{Q}_{M}}\right)$ are orthogonal, and $W^{\mathbb{Q}_{M}}$ is a $\mathbb{Q}_{M}$-Brownian motion. An example where this pertains is given in Monoyios 33, in a two-dimensional model of basis risk with partial information. The indifference price process expansion is given by the analogue of (5.20), as

$$
p_{t}(\alpha)=\mathbb{E}^{\mathbb{Q}_{M}}\left[F \mid \mathcal{F}_{t}\right]+\frac{1}{2} \alpha\left(\operatorname{var}^{\mathbb{Q}_{M}}\left[F \mid \mathcal{F}_{t}\right]-\mathbb{E}^{\mathbb{Q}_{M}}\left[\int_{t}^{T}\left\|\theta_{u}^{M}\right\|^{2} \mathrm{~d}[S]_{u}\right]\right)+O\left(\alpha^{2}\right), \quad 0 \leq t \leq T .
$$

When the model is Markovian, the integrand $\theta^{M}$ can sometimes be expressed in terms of the partial derivatives with respect to $S$ and $Y$ of the marginal price process $\widehat{p}\left(t, S_{t}, Y_{t}\right)=\mathbb{E}^{\mathbb{Q}_{M}}\left[F \mid S_{t}, Y_{t}\right]$. An example where this is carried out can be found in Monoyios 33 .

Example 6.2 (Two-dimensional random parameter basis risk model). This is a random parameter version of the classical example first considered by Davis [10, and so a two-dimensional case of Example 6.1. We show how the asymptotic expansion for the indifference price simplifies in this case, and so we extend results of Davis and others [10, 18, 29, to general (so possibly pathdependent) payoffs dependent on the non-traded asset price, in a random parameter scenario. We also illustrate how the conditions in Assumption 2.1 are satisfied in the case of a lookback put option in the constant parameter (lognormal) case.

Set $d=1, m=2$ in Example 6.1, and set

$$
\beta_{t}=\sigma_{t}^{Y}\left(\rho_{t} \sqrt{1-\rho_{t}^{2}}\right), \quad \rho_{t} \in(-1,1), \quad 0 \leq t \leq T,
$$

for adapted processes $\sigma^{Y}, \rho$. Then the stock and non-traded asset are imperfectly correlated with cross-variation process given by

$$
[S, Y]_{t}=\int_{0}^{t} \rho_{u} \sigma_{u}^{S} \sigma_{u}^{Y} S_{u} Y_{u} \mathrm{~d} u, \quad 0 \leq t \leq T .
$$

The $\mathbb{P}$-dynamics of the assets are

$$
\mathrm{d} S_{t}=\sigma_{t}^{S} S_{t}\left(\lambda_{t}^{S} \mathrm{~d} t+\mathrm{d} W_{t}^{S}\right), \quad \mathrm{d} Y_{t}=Y_{t}\left(\mu_{t}^{Y} \mathrm{~d} t+\sigma_{t}^{Y} \mathrm{~d} W_{t}^{Y}\right), \quad \lambda_{t}^{S}:=\mu_{t}^{S} / \sigma_{t}^{S}, \quad 0 \leq t \leq T,
$$

where $W^{Y}=\rho W^{S}+\sqrt{1-\rho^{2}} W^{S, \perp}$.

The density process of any ELMM $\mathbb{Q} \in \mathbf{M}_{f}$ is once again given by (6.1), with $\lambda_{t}^{S}=\mu_{t}^{S} / \sigma_{t}^{S}$, and in this case the processes in the Doléans exponential are one-dimensional. For the MEMM $\mathbb{Q}^{0} \equiv \mathbb{Q}_{E}$, the integrand $\gamma$ in $(6.1)$ is given by some process $\gamma^{0}$. We may write the $\mathbb{Q}$-dynamics of $Y$ as a perturbation to the $\mathbb{Q}^{0}$-dynamics, in the same manner as in Section 5 . This gives the $\mathbb{Q}$-dynamics of the asset prices in the form

$$
\mathrm{d} S_{t}=\sigma_{t}^{S} S_{t} \mathrm{~d} W_{t}^{S, \mathbb{Q}}, \quad \mathrm{d} Y_{t}=Y_{t}\left[\nu_{t} \mathrm{~d} t+\sigma_{t}^{Y}\left(\mathrm{~d} W_{t}^{Y, \mathbb{Q}}+\sqrt{1-\rho_{t}^{2}} \varepsilon \varphi_{t} \mathrm{~d} t\right)\right]
$$

for $\mathbb{Q}$-Brownian motions $W^{S, \mathbb{Q}}, W^{Y, \mathbb{Q}}$ with instantaneous correlation $\rho$, so that

$$
W^{Y, \mathbb{Q}}=\rho W^{S, \mathbb{Q}}+\sqrt{1-\rho^{2}} W^{S, \perp, \mathbb{Q},}
$$

with $W^{S, \mathbb{Q}}, W^{S, \perp, \mathbb{Q}}$ independent $\mathbb{Q}$-Brownian motions, $\nu:=\mu^{Y}-\sigma^{Y}\left(\rho \lambda^{S}+\sqrt{1-\rho^{2}} \gamma^{0}\right)$ and $\varepsilon \varphi:=-\left(\gamma-\gamma^{0}\right)$, for a small parameter $\varepsilon$ and control process $\varphi$. For $\varepsilon \varphi=0$ we have the dynamics under the MEMM $\mathbb{Q}^{0}$. Once again, the perturbation expansion for the indifference price of a 
claim with payoff $F$ depending on the evolution of $S, Y$ over $[0, T]$ will be of the form (5.19) or, equivalently, (5.20). In the case that $\lambda^{S}$ has no dependence on $Y$, then $\gamma^{0}=0$ and $\mathbb{Q}^{0}=\mathbb{Q}_{M}$.

Another special case arises when $\rho$ is deterministic (say, constant), $\lambda^{S}, \mu^{Y}, \sigma^{Y}$ are adapted to the filtration generated by $W^{Y}$, so depend on the evolution of the non-traded asset price only, and the claim is written on the non-traded asset, so its payoff $F$ also depends only on the evolution of $Y$. (This would also apply in a stochastic volatility model where $Y$ is the process driving the volatility, and then $F$ would be a volatility derivative.) In this case the Kunita-Watanabe decomposition of $F$ under $\mathbb{Q}^{0}$ will be of the special form

$$
F=\mathbb{E}^{\mathbb{Q}^{0}}[F]+\left(\psi^{(0)} \cdot W^{Y, \mathbb{Q}^{0}}\right)_{T},
$$

for some process $\psi^{(0)}$ such that $\left(\psi^{(0)} \cdot W^{Y, \mathbb{Q}^{0}}\right)$ is a $\mathbb{Q}^{0}$-martingale. But we also have the general form (5.17) of this decomposition, which in this case reads as

$$
F=\mathbb{E}^{\mathbb{Q}^{0}}[F]+\left(\theta^{(0)} \cdot S\right)_{T}+\left(\xi^{(0)} \cdot W^{S, \perp, \mathbb{Q}^{0}}\right)_{T},
$$

for integrands $\theta^{(0)}, \xi^{(0)}$ (here, $\theta^{(0)}$ would be the marginal utility-based hedging strategy for the claim).

Equating the representations in (6.6) and (6.7) and in view of (6.4) and (6.5) for the case $\mathbb{Q}=\mathbb{Q}^{0}$, it is easy to see that $\theta^{(0)}, \xi^{(0)}$ are both linearly related to the process $\psi^{(0)}$, through

$$
\theta^{(0)} \sigma^{S} S=\rho \psi^{(0)}, \quad \xi^{(0)}=\sqrt{1-\rho^{2}} \psi^{(0)} .
$$

It is then straightforward to compute that

$$
\operatorname{var}^{\mathbb{Q}^{0}}[F]=\frac{1}{\rho^{2}} \mathbb{E}^{\mathbb{Q}^{0}}\left[\int_{0}^{T}\left(\theta_{t}^{(0)}\right)^{2} \mathrm{~d}[S]_{t}\right] .
$$

The time-zero indifference price expansion in this case then simplifies to

$$
p_{0}(\alpha)=\mathbb{E}^{\mathbb{Q}^{0}}[F]+\frac{1}{2} \alpha\left(1-\rho^{2}\right) \operatorname{var}^{\mathbb{Q}^{0}}[F]+O\left(\alpha^{2}\right),
$$

which is an extension of the form found in [10, 18, 29] to European payoffs $F$ satisfying Assumption 2.1. in models with random parameters dependent on $Y$. If, in addition, $\lambda^{S}$ is deterministic, then $\mathbb{Q}^{0}=\mathbb{Q}_{M}$.

It is instructive to see how Assumption 2.1 would be checked in a simple case of this example. Suppose the parameters of the model are constants, so that $Y$ is a geometric Brownian motion. Let the claim be a European floating strike lookback put option on the non-traded asset, so that $F$ is a functional of a one-dimensional Brownian motion given by

$$
F=\max _{0 \leq t \leq T} Y_{t}-Y_{T},
$$

To ease notation, write $W \equiv W^{Y, \mathbb{Q}}$ for the Brownian motion driving $Y$ under any ELMM. When the perturbation $\varepsilon \varphi$ is zero, $Y$ satisfies

$$
\mathrm{d} Y_{t}=Y_{t}\left(\nu \mathrm{d} t+\eta \mathrm{d} W_{t}\right), \quad Y_{0}=y \in \mathbb{R}^{+},
$$

for constants $\nu$ and $\eta>0$. For concreteness, let us suppose that $\nu-\frac{1}{2} \eta^{2}>0$. The functional $F \equiv F(W)$ is given by

$$
F(W)=y \exp \left[\left(\nu-\frac{1}{2} \eta^{2}\right) T\right]\left(\exp \left(\eta \max _{0 \leq t \leq T} W_{t}\right)-\exp \left(\eta W_{T}\right)\right) .
$$

Consider the two functionals $F_{1}(W):=\exp \left(\eta W_{T}\right)$ and $F_{2}(W):=\exp \left(\eta \max _{0 \leq t \leq T} W_{t}\right)$ in turn.

For $F_{1}$, it is straightforward to see square-integrability, and that Assumption 2.1(ii) is satisfied with $k=F 1$ and $g(\varepsilon)=\exp (\eta \varepsilon)-1$. It is also easy to compute

$$
\lim _{\varepsilon \rightarrow 0} \frac{1}{\varepsilon}\left[F_{1}\left(W+\varepsilon \int_{0}^{.} \varphi_{s} \mathrm{~d} s\right)-F_{1}(W)\right]=\eta Y_{T} \int_{0}^{T} \varphi_{t} \mathrm{~d} t=\int_{0}^{T} \eta F_{1}(W) \varphi_{t} \mathrm{~d} t .
$$

Therefore, $\partial F_{1}(W ;(t, T])=\eta F_{1}(W)$. 
For $F_{2}$, the maximum of the Brownian motion over $[0, T]$ is achieved at some random time $\tau(\omega) \equiv \tau(W)$, so in this case we have

$$
F_{2}(W)=y \exp \left[\left(\nu-\frac{1}{2} \eta^{2}\right) T+\eta W_{\tau(W)}\right] .
$$

The first two conditions in Assumption 2.1 are satisfied in a similar manner as for $F_{1}$. For the last condition, with $\Phi=\int_{0}^{\cdot} \varphi_{s} \mathrm{~d} s$ we obtain

$$
\lim _{\varepsilon \rightarrow 0} \frac{1}{\varepsilon}\left[F_{2}(W+\varepsilon \Phi)-F_{2}(W)\right]=\eta F_{2}(W) \Phi_{\tau(W)}=\int_{0}^{T} \eta F_{2}(W) \mathbb{1}_{\{\tau(W)>t\}} \varphi_{t} \mathrm{~d} t .
$$

Therefore, $\partial F_{2}(W ;(t, T])=\eta F_{2}(W) \mathbb{1}_{\{\tau(W)>t\}}$. This shows how Assumption 2.1 is compatible with path-dependent payoffs. Similar reasoning can work with random parameter models.

Example 6.3 (Basis risk with stochastic correlation). This model has been considered by Ankirchner and Heyne [1, who examined local risk minimisation methods for hedging basis risk. A traded asset $S$ and non-traded asset $Y$ follow correlated geometric Brownian motions, as in Example 6.2, but the correlation $\rho=\left(\rho_{t}\right)_{0 \leq t \leq T}$ is now stochastic. In this case, we have $m=3$, $d=1$. With $W$ a three-dimensional Brownian motion, let $W^{S}=W^{1}, W^{Y}=\rho W^{1}+\sqrt{1-\rho^{2}} W^{2}$, $W^{\rho}=\delta W^{1}+\eta W^{2}+\sqrt{1-\delta^{2}-\eta^{2}} W^{3}$, for constants $\delta, \eta$ such that $\delta^{2}+\eta^{2} \leq 1$. The state variable dynamics are then

$$
\begin{aligned}
\mathrm{d} S_{t} & =\sigma^{S} S_{t}\left(\lambda^{S} \mathrm{~d} t+\mathrm{d} W_{t}^{1}\right), \\
\mathrm{d} Y_{t} & =Y_{t}\left[\mu^{Y} \mathrm{~d} t+\sigma^{Y}\left(\rho_{t} \mathrm{~d} W_{t}^{1}+\sqrt{1-\rho_{t}^{2}} \mathrm{~d} W_{t}^{2}\right)\right], \\
\mathrm{d} \rho_{t} & =g_{t} \mathrm{~d} t+h_{t}\left(\delta \mathrm{d} W_{t}^{1}+\eta \mathrm{d} W_{t}^{2}+\sqrt{1-\delta^{2}-\eta^{2}} \mathrm{~d} W_{t}^{3}\right) .
\end{aligned}
$$

Here, $g, h$ are adapted processes such that $\rho_{t} \in[-1,1]$ almost surely. Ankirchner and Heyne [1] give some specific examples of such models.

In this example we also have $\mathbb{Q}^{0}=\mathbb{Q}_{M}$, with $Z^{\mathbb{Q}_{M}}=\mathcal{E}\left(-\lambda^{S} W^{1}\right)$, and the Föllmer-SchweizerSondermann decomposition of the claim is of the form

$$
F=\mathbb{E}^{\mathbb{Q}_{M}}[F]+\left(\theta^{M} \cdot S\right)_{T}+\left(\xi^{M} \cdot W^{2, \mathbb{Q}_{M}}\right)_{T}+\left(\phi^{M} \cdot W^{3, \mathbb{Q}_{M}}\right)_{T}
$$

for some integrands $\theta^{M}, \xi^{M}, \phi^{M}$, where $W^{\mathbb{Q}_{M}}=\left(W^{1, \mathbb{Q}_{M}}, W^{2, \mathbb{Q}_{M}}, W^{3, \mathbb{Q}_{M}}\right)^{\top}$ is a three-dimensional $\mathbb{Q}_{M}$-Brownian motion, the first of which drives the stock, so that the stochastic integrals in (6.8) are orthogonal $\mathbb{Q}_{M}$-martingales. The time-zero indifference price expansion is again of the form (6.3).

Many examples are covered by the framework of Theorem [5.1, including classical stochastic volatility models, basis risk models with stochastic volatility (so $m=3, d=1$ ) with a traded and non-traded asset both driven by a common stochastic volatility process (and stochastic correlation can be added to this framework), or basis risk models with unknown asset drifts, extending [33. (which modelled the drifts as unknown constants) to model the drifts as linear diffusions.

6.1. Entropy minimisation in stochastic volatility models. We end with another application of the asymptotic methods developed in the paper. This time, we are interested in finding the minimal entropy martingale measure $\mathbb{Q}^{0} \equiv \mathbb{Q}_{E}$ in a stochastic volatility model. A traded asset $S$ and a non-traded stochastic factor $Y$ follow, under the physical measure $\mathbb{P}$,

$$
\begin{aligned}
\mathrm{d} S_{t} & =\sigma\left(Y_{t}\right) S_{t}\left(\lambda\left(Y_{t}\right) \mathrm{d} t+\mathrm{d} W_{t}^{S}\right), \\
\mathrm{d} Y_{t} & =a\left(Y_{t}\right) \mathrm{d} t+b\left(Y_{t}\right) \mathrm{d} W_{t}^{Y},
\end{aligned}
$$

for suitable functions $\sigma, \lambda, a, b$ such that there are unique strong solutions to (6.9]6.10). The Brownian motions $W^{S}, W^{Y}$ have constant correlation $\rho \in[-1,1]$. We write $W_{t}^{Y}=\rho W_{t}^{S}+$ $\sqrt{1-\rho^{2}} W_{t}^{S, \perp}$. The density process of any ELMM $\mathbb{Q}$ is

$$
Z_{t}^{\mathbb{Q}}=\mathcal{E}\left(-\lambda \cdot W^{S}-\gamma \cdot W^{S, \perp}\right)_{t}, \quad 0 \leq t \leq T,
$$


for some square-integrable process $\gamma$ such that $Z^{\mathbb{Q}}$ is a $\mathbb{P}$-martingale.

The entropy minimisation problem is the stochastic control problem to minimise

$$
I_{0}(\mathbb{Q} \mid \mathbb{P})=\mathbb{E}^{\mathbb{Q}}\left[\frac{1}{2} \int_{0}^{T}\left(\lambda^{2}\left(Y_{t}\right)+\gamma_{t}^{2}\right) \mathrm{d} t\right]
$$

over control processes $\gamma$, where we assume that $I_{0}(\mathbb{Q} \mid \mathbb{P})<\infty$, and where, under $\mathbb{Q}, S, Y$ follow

$$
\begin{aligned}
\mathrm{d} S_{t} & =\sigma\left(Y_{t}\right) S_{t} \mathrm{~d} W_{t}^{S, \mathbb{Q}}, \\
\mathrm{d} Y_{t} & =\left(a\left(Y_{t}\right)-b\left(Y_{t}\right) \rho \lambda\left(Y_{t}\right)\right) \mathrm{d} t+b\left(Y_{t}\right)\left(\mathrm{d} W_{t}^{Y, \mathbb{Q}}-\sqrt{1-\rho^{2}} \gamma_{t} \mathrm{~d} t\right),
\end{aligned}
$$

for $\mathbb{Q}$-Brownian motions $W^{S, \mathbb{Q}}, W^{Y, \mathbb{Q}}$ with correlation $\rho$, such that setting $\gamma=0$ yields the minimal martingale measure $\mathbb{Q}_{M}$.

The idea here is to consider the drift adjustment $\sqrt{1-\rho^{2}} \gamma_{t}$ in (6.11) as a perturbation to the Brownian paths, and hence to convert the entropy minimisation problem to the type of control problem we have considered in Section 3, in the limit that the absolute value of the correlation is close to 1 , so $1-\rho^{2}$ is small. To this end, we define a parameter $\varepsilon$ and a control process $\varphi$ such that

$$
\varepsilon^{2}=1-\rho^{2}, \quad \varepsilon \varphi=-\sqrt{1-\rho^{2}} \gamma,
$$

and we define a parametrised family of measures $\{\mathbb{Q}(\varepsilon)\}_{\varepsilon \in \mathbb{R}}$, such that

$$
\mathbb{Q}=\mathbb{Q}(\varepsilon), \quad \mathbb{Q}(0)=\mathbb{Q}_{M}
$$

The state variable dynamics for $Y$ are then given by

$$
\mathrm{d} Y_{t}=\left(a\left(Y_{t}\right)-b\left(Y_{t}\right) \rho \lambda\left(Y_{t}\right)\right) \mathrm{d} t+b\left(Y_{t}\right)\left(\mathrm{d} W_{t}^{Y, \mathbb{Q}(\varepsilon)}+\varepsilon \varphi_{t} \mathrm{~d} t\right)
$$

With $\Phi:=\int_{0}^{\cdot} \varphi_{s} \mathrm{~d} s$, we define a square-integrable functional $F \equiv F\left(W^{\mathbb{Q}(\varepsilon)}+\varepsilon \Phi\right)$ of the Brownian paths by

$$
F:=\frac{1}{2} \int_{0}^{T} \lambda^{2}\left(Y_{t}\right) \mathrm{d} t=: \frac{1}{2} K_{T},
$$

where, for brevity of notation, we have defined the so-called mean-variance trade-off process $K$ by

$$
K_{t}:=\int_{0}^{t} \lambda^{2}\left(Y_{u}\right) \mathrm{d} u, \quad 0 \leq t \leq T .
$$

We assume that the model is such that $K_{T}$ defines a functional satisfying Assumption 2.1],

In this notation, the relative entropy between the minimal martingale measure and $\mathbb{P}$ is

$$
I_{0}\left(\mathbb{Q}_{M} \mid \mathbb{P}\right)=\mathbb{E}^{\mathbb{Q}_{M}}\left[\frac{1}{2} K_{T}\right]=\mathbb{E}^{\mathbb{Q}(0)}\left[F\left(W^{\mathbb{Q}(0)}\right)\right] .
$$

The control problem to minimise $I_{0}(\mathbb{Q} \mid \mathbb{P})$ over ELMMs $\mathbb{Q} \in \mathbf{M}_{f}$ then has value function

$$
I_{0}\left(\mathbb{Q}_{E} \mid \mathbb{P}\right):=\inf _{\varphi \in \mathcal{A}\left(\mathbf{M}_{f}\right)} \mathbb{E}^{\mathbb{Q}(\varepsilon)}\left[F\left(W^{\mathbb{Q}(\varepsilon)}+\varepsilon \int_{0}^{\cdot} \varphi_{s} \mathrm{~d} s\right)+\frac{1}{2} \int_{0}^{T} \varphi_{t}^{2} \mathrm{~d} t\right],
$$

where $\mathcal{A}\left(\mathbf{M}_{f}\right)$ denotes the set of controls $\varphi$ such that $I_{0}(\mathbb{Q} \mid \mathbb{P})$ is finite.

We have now formulated the entropy minimisation problem in the form we need to be able to apply the Malliavin asymptotic method, and this gives the theorem below.

Theorem 6.4. In the stochastic volatility model defined by (6.9][6.10), suppose the terminal value $K_{T}$ of mean-variance trade-off process in (6.13) defines a Brownian functional satisfying Assummption 2.1. Then the relative entropy between the minimal entropy martingale measure $\mathbb{Q}_{E}$ and $\mathbb{P}$, in the limit that $1-\rho^{2}$ is close to 1 , is given as

$$
I_{0}\left(\mathbb{Q}_{E} \mid \mathbb{P}\right)=I_{0}\left(\mathbb{Q}_{M} \mid \mathbb{P}\right)-\frac{1}{8}\left(1-\rho^{2}\right) \operatorname{var}^{\mathbb{Q}_{M}}\left[K_{T}\right]+O\left(\left(1-\rho^{2}\right)^{2}\right)
$$

where $\mathbb{Q}_{M}$ is the minimal martingale measure. 
Proof. This is along the same lines as previous results, so we only sketch the details. One appeals to the decomposition of $F$ under $\mathbb{Q}(0)$, which is of the form

$$
F\left(W^{\mathbb{Q}(0)}\right)=\mathbb{E}^{\mathbb{Q}(0)}\left[F\left(W^{\mathbb{Q}(0)}\right)\right]+\left(\xi^{(0)} \cdot W^{\mathbb{Q}(0)}\right)_{T},
$$

for some integrand $\xi^{(0)}$. Such a decomposition exists uniquely, given that $F$ depends only on $Y$, and the dynamics in (6.12). We expand the objective function (6.15) about $\varepsilon=0$ and use the representation (6.16). This gives

$$
\begin{aligned}
& \mathbb{E}^{\mathbb{Q}(\varepsilon)}\left[F\left(W^{\mathbb{Q}(\varepsilon)}+\varepsilon \int_{0}^{.} \varphi_{s} \mathrm{~d} s\right)+\frac{1}{2} \int_{0}^{T} \varphi_{t}^{2} \mathrm{~d} t\right] \\
= & \mathbb{E}^{\mathbb{Q}(0)}\left[F\left(W^{\mathbb{Q}(0)}\right)+\int_{0}^{T}\left(\varepsilon \xi_{t}^{(0)} \varphi_{t}+\frac{1}{2} \varphi_{t}^{2}\right) \mathrm{d} t\right]+o(\varepsilon) .
\end{aligned}
$$

We minimise the right-hand-side over $\varphi$ by choosing $\varphi=-\varepsilon \xi^{(0)}$. Using (6.16) again, and recalling (6.14), the result follows.

Remark 6.5. In 31, 30], Esscher transform relations between $\mathbb{Q}_{E}$ and $\mathbb{Q}_{M}$ are derived, and it is an exercise in asymptotic analysis to see that those results are consistent with Theorem 6.4.

\section{Conclusions}

It is quite natural to apply Malliavin calculus ideas in stochastic control problems where the control turns out to be a drift which is considered as a perturbation to a Brownian motion, and this is the path taken in this paper. We have shown how the method can yield small risk aversion asymptotic expansions for exponential indifference prices in Itô process models, and how one can identify the minimal entropy measure as a perturbation to the minimal martingale measure in stochastic volatility models. It would be interesting to extend the method to models with jumps in the underlying state process.

\section{REFERENCES}

[1] Stefan Ankirchner and Gregor Heyne, Cross hedging with stochastic correlation, Finance Stoch., 16 (2012), pp. 17-43.

[2] DIRK BECHERER, Rational hedging and valuation of integrated risks under constant absolute risk aversion, Insurance Math. Econom., 33 (2003), pp. 1-28.

[3] E. Benhamou, E. Gobet, And M. Miri, Time dependent Heston model, SIAM J. Financial Math., 1 (2010), pp. 289-325.

[4] Sara Biagini And Marco Frittelli, The supermartingale property of the optimal wealth process for general semimartingales, Finance Stoch., 11 (2007), pp. 253-266.

[5] Joris Bierkens ANd Hilbert J. KAPPEn, Explicit solution of relative entropy weighted control. Preprint, available at http://arxiv.org/pdf/1205.6946v3.pdf, 2012.

[6] Jean-Michel Bismut, Martingales, the Malliavin calculus and hypoellipticity under general Hörmander's conditions, Z. Wahrsch. Verw. Gebiete, 56 (1981), pp. 469-505.

[7] Michelle Boué and Paul Dupuis, A variational representation for certain functionals of Brownian motion, Ann. Probab., 26 (1998), pp. 1641-1659.

[8] J. M. C. CLARK, The representation of functionals of Brownian motion by stochastic integrals, Ann. Math. Statist., 41 (1970), pp. 1282-1295.

[9] Mark H. A. Davis, Option pricing in incomplete markets, in Mathematics of derivative securities (Cambridge, 1995), vol. 15 of Publ. Newton Inst., Cambridge Univ. Press, Cambridge, 1997, pp. 216-226.

[10] _ - Optimal hedging with basis risk, in From stochastic calculus to mathematical finance, Y. Kabanov, R. Liptser, and J. Stoyanov, eds., Springer, Berlin, 2006, pp. 169-187.

[11] Freddy Delbaen, Peter Grandits, Thorsten Rheinländer, Dominick Samperi, Martin Schweizer, And Christophe Stricker, Exponential hedging and entropic penalties, Math. Finance, 12 (2002), pp. 99-123.

[12] Freddy Delbaen And Walter Schachermayer, A general version of the fundamental theorem of asset pricing, Math. Ann., 300 (1994), pp. 463-520.

[13] Hans Föllmer and Dieter Sondermann, Hedging of nonredundant contingent claims, in Contributions to mathematical economics, North-Holland, Amsterdam, 1986, pp. 205-223. 
[14] Jean-Pierre Fouque, George Papanicolaou, Ronnie Sircar, and Knut Sølna, Multiscale stochastic volatility for equity, interest rate, and credit derivatives, Cambridge University Press, Cambridge, 2011.

[15] Eric Fournié, Jean-Michel Lasry, Jérôme Lebuchoux, Pierre-Louis Lions, and Nizar Touzi, Applications of Malliavin calculus to Monte Carlo methods in finance, Finance Stoch., 3 (1999), pp. 391-412.

[16] Marco Frittelli, The minimal entropy martingale measure and the valuation problem in incomplete markets, Math. Finance, 10 (2000), pp. 39-52.

[17] Peter Grandits and Thorsten Rheinländer, On the minimal entropy martingale measure, Ann. Probab., 30 (2002), pp. 1003-1038.

[18] Vicky Henderson, Valuation of claims on nontraded assets using utility maximization, Math. Finance, 12 (2002), pp. 351-373.

[19] Vicky Henderson and Gechun Liang, A multidimensional exponential utility indifference pricing model with applications to counterparty risk. Preprint, available at http://arxiv.org/pdf/1111.3856v2.pdf, 2012.

[20] Peter Imkeller, Malliavin's calculus in insider models: additional utility and free lunches, Math. Finance, 13 (2003), pp. 153-169. Conference on Applications of Malliavin Calculus in Finance (Rocquencourt, 2001).

[21] Yuri M. Kabanov And Christophe Stricker, On the optimal portfolio for the exponential utility maximization: remarks to the six-author paper, Math. Finance, 12 (2002), pp. 125-134.

[22] Jan Kallsen, Johannes Muhle-Karbe, and Richard Vierthauer, Asymptotic power utility-based pricing and hedging, Mathematics and Financial Economics, (2013). in press; doi:10.1007/s11579-013-0095-8.

[23] Jan KAllsen And Thorsten Rheinländer, Asymptotic utility-based pricing and hedging for exponential utility, Statist. Decisions, 28 (2011), pp. 17-36.

[24] Ioannis Karatzas and Steven E. Shreve, Methods of mathematical finance, vol. 39 of Applications of Mathematics (New York), Springer-Verlag, New York, 1998.

[25] D. Kramkov And M. SîRBu, Asymptotic analysis of utility-based hedging strategies for small number of contingent claims, Stochastic Process. Appl., 117 (2007), pp. 1606-1620.

[26] Tim Leung, Ronnie Sircar, and Thaleia Zariphopoulou, Forward indifference valuation of American options, Stochastics, 84 (2012), pp. 741-770.

[27] David G. Luenberger, Optimization by vector space methods, John Wiley \& Sons Inc., New York, 1969.

[28] Michael Mania and Martin Schweizer, Dynamic exponential utility indifference valuation, Ann. Appl. Probab., 15 (2005), pp. 2113-2143.

[29] Michael Monoyios, Performance of utility-based strategies for hedging basis risk, Quant. Finance, 4 (2004), pp. 245-255.

[30] - Characterisation of optimal dual measures via distortion, Decis. Econ. Finance, 29 (2006), pp. 95-119.

[31] — The minimal entropy measure and an Esscher transform in an incomplete market model, Statist. Probab. Lett., 77 (2007), pp. 1070-1076.

[32] — Optimal hedging and parameter uncertainty, IMA J. Manag. Math., 18 (2007), pp. 331-351.

[33] - Utility-based valuation and hedging of basis risk with partial information, Applied Mathematical Finance, 17 (2010), pp. 519-551.

[34] David Nualart, The Malliavin calculus and related topics, Probability and its Applications (New York), Springer-Verlag, Berlin, second ed., 2006.

[35] Daniel Revuz And Marc Yor, Continuous martingales and Brownian motion, vol. 293 of Grundlehren der Mathematischen Wissenschaften [Fundamental Principles of Mathematical Sciences], Springer-Verlag, Berlin, third ed., 1999.

[36] L. C. G. Rogers and David Williams, Diffusions, Markov processes, and martingales. Vol. 2, Cambridge Mathematical Library, Cambridge University Press, Cambridge, 2000. Itô calculus, Reprint of the second (1994) edition.

[37] Walter Schachermayer, Optimal investment in incomplete markets when wealth may become negative, Ann. Appl. Probab., 11 (2001), pp. 694-734.

[38] — - A super-martingale property of the optimal portfolio process, Finance Stoch., 7 (2003), pp. 433-456.

[39] Ronnie Sircar and Thaleia Zariphopoulou, Bounds and asymptotic approximations for utility prices when volatility is random, SIAM J. Control Optim., 43 (2004/05), pp. 1328-1353 (electronic).

[40] Christophe Stricker, Indifference pricing with exponential utility, in Seminar on Stochastic Analysis, Random Fields and Applications IV, vol. 58 of Progr. Probab., Birkhäuser, Basel, 2004, pp. 323-328.

[41] Arthur Wouk, A course of applied functional analysis, Wiley-Interscience [John Wiley \& Sons], New York, 1979. Pure and Applied Mathematics.

[42] Thaleia Zariphopoulou, A solution approach to valuation with unhedgeable risks, Finance Stoch., 5 (2001), pp. $61-82$.

Michael Monoyios, Mathematical Institute, University of Oxford, Radcliffe Observatory QuarTER, WoOdstock RoAd, OXford OX2 6GG, UK

E-mail address: monoyios@maths.ox.ac.uk 